TRANSACTIONS OF THE

AMERICAN MATHEMATICAL SOCIETY

Volume 363, Number 12, December 2011, Pages 6505-6529

S 0002-9947(2011)05305-5

Article electronically published on July 25, 2011

\title{
SYSTEMS FORMED BY TRANSLATES OF ONE ELEMENT IN $L_{p}(\mathbb{R})$
}

\author{
E. ODELL, B. SARI, TH. SCHLUMPRECHT, AND B. ZHENG
}

\begin{abstract}
Let $1 \leq p<\infty, f \in L_{p}(\mathbb{R})$, and $\Lambda \subseteq \mathbb{R}$. We consider the closed subspace of $L_{p}(\mathbb{R}), X_{p}(f, \Lambda)$, generated by the set of translations $f_{(\lambda)}$ of $f$ by $\lambda \in \Lambda$. If $p=1$ and $\left\{f_{(\lambda)}: \lambda \in \Lambda\right\}$ is a bounded minimal system in $L_{1}(\mathbb{R})$, we prove that $X_{1}(f, \Lambda)$ embeds almost isometrically into $\ell_{1}$. If $\left\{f_{(\lambda)}: \lambda \in \Lambda\right\}$ is an unconditional basic sequence in $L_{p}(\mathbb{R})$, then $\left\{f_{(\lambda)}: \lambda \in \Lambda\right\}$ is equivalent to the unit vector basis of $\ell_{p}$ for $1 \leq p \leq 2$ and $X_{p}(f, \Lambda)$ embeds into $\ell_{p}$ if $2<p \leq 4$. If $p>4$, there exists $f \in L_{p}(\mathbb{R})$ and $\Lambda \subseteq \mathbb{Z}$ so that $\left\{f_{(\lambda)}: \lambda \in \Lambda\right\}$ is unconditional basic and $L_{p}(\mathbb{R})$ embeds isomorphically into $X_{p}(f, \Lambda)$.
\end{abstract}

\section{INTRODUCTION}

Let $f: \mathbb{R} \rightarrow \mathbb{R}$ and $\lambda \in \mathbb{R}$. We denote by $f_{(\lambda)}$ the translation of $f \lambda$-units to the right for $\lambda>0$ (and $|\lambda|$-units to the left for $\lambda<0$ ). Precisely,

$$
f_{(\lambda)}(x)=f(x-\lambda) \text { for } x \in \mathbb{R} .
$$

If $f \in L_{p}(\mathbb{R}), 1 \leq p<\infty$ and $\Lambda \subseteq \mathbb{R}$, we let $X_{p}(f, \Lambda)$ equal $\left[\left\{f_{(\lambda)}: \lambda \in \Lambda\right\}\right]$, where [.] denotes the closed linear span in $L_{p}(\mathbb{R})$. Our main focus shall be on the nature of such subspaces given that $\left\{f_{(\lambda)}: \lambda \in \Lambda\right\}$ has some additional structure and $\Lambda$ is uniformly discrete, i.e.,

$$
\inf \left\{\left|\lambda-\lambda^{\prime}\right|: \lambda, \lambda^{\prime} \in \Lambda, \lambda \neq \lambda^{\prime}\right\}>0 .
$$

The "additional structure" takes several forms: $\left\{f_{(\lambda)}: \lambda \in \Lambda\right\}$ is a bounded minimal system or is unconditional basic or can be ordered to be a (Schauder) basis or a (Schauder) frame for $X_{p}(f, \Lambda)$. It is worth mentioning that it is known that if $\left\{f_{(\lambda)}: \lambda \in \Lambda\right\}$ is a bounded minimal system, in particular, if it can be ordered to be basic, then $\Lambda$ must be uniformly discrete. This is easy (Proposition 1.8 below).

The nature of $X_{p}(f, \Lambda)$ and $\left\{f_{(\lambda)}: \lambda \in \Lambda\right\}$ have been studied in a number of papers, mainly using techniques of harmonic analysis. Our techniques will be, largely, from the geometry of Banach spaces. We recall seven theorems, beginning with Wiener's famous Tauberian theorem.

Theorem 1.1 (Wi $)$. For $f \in L_{2}(\mathbb{R}), X_{2}(f, \mathbb{R})=L_{2}(\mathbb{R})$ if and only if $\hat{f}(t) \neq 0$ a.e. For $f \in L_{1}(\mathbb{R}), X_{1}(f, \mathbb{R})=L_{1}(\mathbb{R})$ if and only if $\hat{f}(t) \neq 0$ for all $t \in \mathbb{R}$.

Received by the editors June 10, 2009 and, in revised form, December 25, 2009.

2010 Mathematics Subject Classification. Primary 42C30, 46E30; Secondary 46B15.

The first, third, and fourth authors were partially supported by National Science Foundation Grants DMS-0968813 \& DMS-0700126, DMS-0856148 \& DMS-0556013, and DMS-1068838, respectively.

(C)2011 American Mathematical Society Reverts to public domain 28 years from publication 
Theorem 1.2 ([AO Theorem 2.1]). Let $2<p<\infty$. There exists $f \in L_{p}(\mathbb{R})$, all of whose derivatives exist and are in $L_{2}(\mathbb{R})$ (i.e., $f \in H^{2, \infty}(\mathbb{R})$ ) so that $X_{p}(f, \mathbb{Z})=$ $L_{p}(\mathbb{R})$. Moreover, $f$ can be chosen to satisfy, in addition, any one of the following conditions:

(1) $X_{p}(f, \mathbb{N} \cup\{0\})=L_{p}(\mathbb{R})$.

(2) $\left(f_{(n)}\right)_{n \in \mathbb{Z}}$ is orthogonal in $L_{2}(\mathbb{R})$.

(3) $\left(f_{(n)}\right)_{n \in \mathbb{Z}}$ is a bounded minimal system.

Theorem 1.3 ([AO]). Let $1 \leq p \leq 2$, and let $F \subseteq L_{p}(\mathbb{R})$ be a finite set. Then $\left[\left\{f_{(n)}: f \in F, n \in \mathbb{Z}\right\}\right] \neq L_{p}(\mathbb{R})$.

Theorem 1.4 ([ER, Corollary 2.11]). Let $1 \leq p<\infty, 0 \neq f \in L_{p}(\mathbb{R})$. Then $\left\{f_{(\lambda)}: \lambda \in \mathbb{R}\right\}$ is linearly independent.

Theorem 1.5 ([O]]). Let $\Lambda=\left\{\lambda_{n}\right\}_{n \in \mathbb{Z}} \subseteq \mathbb{R}$ so that $\Lambda \cap \mathbb{Z}=\emptyset$ and $\lim _{|n| \rightarrow \infty}\left|\lambda_{n}-n\right|$ $=0$. Then there exists $f \in L_{2}(\mathbb{R})$ so that $X_{2}(f, \Lambda)=L_{2}(\mathbb{R})$.

Theorem 1.6 ([OZ, Theorem 2]). There is no unconditional basis of translates of $f,\left\{f_{(\lambda)}: \lambda \in \Lambda\right\}$, with $X_{2}(f, \Lambda)=L_{2}(\mathbb{R})$.

So the space $X_{p}(f, \Lambda), \Lambda$ uniformly discrete, can equal $L_{p}(\mathbb{R})$, for $p \geq 2$ at least. For $p=1$ the situation is different as pointed out to us by J. Bruna.

Theorem 1.7. Let $f \in L_{1}(\mathbb{R})$, and let $\Lambda \subseteq \mathbb{R}$ be uniformly discrete. Then $X(f, \Lambda) \neq L_{1}(\mathbb{R})$.

This seems to be a folklore theorem and we were unable to find a reference. It follows from Theorem 1.1 and Lemma 3.3 below (and can also be deduced from BOU] and the proof of Lemma 3.3).

For $1<p<\infty$ it remains an open problem whether there exists $\Lambda \subseteq \mathbb{R}$ and $f \in L_{p}(\mathbb{R})$ so that, in some order, $\left\{f_{(\lambda)}: \lambda \in \Lambda\right\}$ is a basis for $L_{p}(\mathbb{R})$.

In section 2 we prove that if $f \in L_{1}(\mathbb{R})$ and $\left\{f_{(\lambda)}: \lambda \in \Lambda\right\}$ is a bounded minimal system for $X_{1}(f, \Lambda)$, then $X_{1}(f, \Lambda)$ embeds almost isometrically into $\ell_{1}$. The same conclusion holds if $\Lambda$ is uniformly discrete and $\left\{f_{(\lambda)}: \lambda \in \Lambda\right\}$ can be ordered to be a (Schauder) frame for $X_{1}(f, \Lambda)$.

In Corollary 2.10 we show that for $1 \leq p \leq 2$, if $\left(f_{(\lambda)}\right)_{\lambda \in \Lambda}$ is an unconditional basic sequence, then $\left(f_{(\lambda)}\right)_{\lambda \in \Lambda}$ is equivalent to the unit vector basis of $\ell_{p}$. For $2<p \leq 4$, we show (Theorem 2.11) that if $\left(f_{(\lambda)}\right)_{\lambda \in \Lambda}$ is unconditional basic, then $X_{p}(f, \Lambda)$ embeds isomorphically into $\ell_{p}$ but (Proposition 2.15) $\left(f_{(\lambda)}\right)_{\lambda \in \Lambda}$ need not be equivalent to the unit vector basis of $\ell_{p}$. For $4<p<\infty$, we give an example (Theorem 2.14) of an unconditional basic sequence $\left(f_{(\lambda)}\right)_{\lambda \in \Lambda}$ so that $X_{p}(f, \Lambda)$ contains an isomorph of $L_{p}[0,1]$ (which, of course, is isometric to $L_{p}(\mathbb{R})$ ).

Among further results in section 2, we also consider the translation problem for the translation invariant space $L_{p}(\mathbb{R}) \cap L_{2}(\mathbb{R}), 2<p<\infty$, and show (Proposition 2.18) that if $\left(f_{(\lambda)}\right)_{\lambda \in \Lambda}$ is unconditional basic, then it is equivalent to the unit vector basis of $\ell_{2}$.

In the beginning of section 3 , we revisit the problem for integer translates of $f \in L_{p}(\mathbb{R})$. We also prove that if $f \in L_{1}(\mathbb{R})$ with $\hat{f}(t) \neq 0$ for all $t$, then $X_{1}(f, \mathbb{Z})$ embeds into $\ell_{1}$ (Proposition 3.4). We also consider discrete versions of our problem for $\ell_{p}(\mathbb{Z}, X)$ in Propositions 3.5 and 3.7 and Corollary 3.12. Fourier analysis plays a role in some of these results. 
In section 4, we recall some additional known results from the literature and list some remaining open problems.

We use standard Banach space notation as may be found in [LT] or [JL. Background material on bases, unconditional bases and such can be found there. For the benefit of those less familiar with these notions, we recall some definitions and facts. A biorthogonal system is a sequence $\left(x_{i}, x_{i}^{*}\right)_{i=1}^{\infty} \subseteq X \times X^{*}$ where $x_{i}^{*}\left(x_{j}\right)=\delta_{(i, j)}$. A biorthogonal system $\left(x_{i}, x_{i}^{*}\right)_{i=1}^{\infty} \subseteq X \times X^{*}$ is fundamental (or complete) if $\left[\left(x_{i}\right)_{i \in \mathbb{N}}\right]=X$ and bounded if $\sup _{i}\left\|x_{i}\right\|\left\|x_{i}^{*}\right\|<\infty$.

$\left(x_{i}\right)_{i=1}^{\infty} \subseteq X$ is a minimal system if there exists $\left(x_{i}^{*}\right)_{i=1}^{\infty} \subseteq X^{*}$ so that $\left(x_{i}, x_{i}^{*}\right)_{i=1}^{\infty}$ is a biorthogonal system. This is equivalent to $x_{i} \notin\left[x_{j}: j \neq i\right]$ for all $i \in \mathbb{N}$. $\left(x_{i}\right)_{i=1}^{\infty}$ is a bounded minimal system if, in addition, $\left(x_{i}, x_{i}^{*}\right)_{i=1}^{\infty}$ is a bounded biorthogonal system. This is equivalent to $\inf _{i} \operatorname{dist}\left(x_{i},\left[x_{j}: j \neq i\right]\right)>0 .\left(x_{i}\right)_{i=1}^{\infty} \subseteq X$ is a (Schauder) basis for $X$ if for all $x \in X$ there exists a unique sequence of scalars $\left(a_{i}\right)_{i=1}^{\infty}$ so that $x=\sum_{i=1}^{\infty} a_{i} x_{i}$. This is equivalent to saying that all $x_{i} \neq 0,\left[\left(x_{i}\right)\right]=$ $X$ and for some $K<\infty$, all $m<n$ in $\mathbb{N}$ and all $\left(a_{i}\right)_{1}^{n} \subseteq \mathbb{R},\left\|\sum_{i=1}^{m} a_{i} x_{i}\right\| \leq$ $K\left\|\sum_{i=1}^{n} a_{i} x_{i}\right\|$. The smallest such $K$ is the basis constant of $\left(x_{i}\right)$. A basis $\left(x_{i}\right)_{i=1}^{\infty}$ for $X$ is a fundamental bounded minimal system for $X$. In this case every $x \in X$ can be written uniquely as $x=\sum_{i=1}^{\infty} x_{i}^{*}(x) x_{i}$. The $x_{i}^{*}$ 's are a basic sequence in $X^{*}$, i.e., form a basis for $\left[\left(x_{i}^{*}\right)\right] \subseteq X^{*}$, and are a basis for $X^{*}$ if $X$ is reflexive. $\left(x_{i}\right)_{i=1}^{\infty}$ is an unconditional basis for $X$ if for all $x \in X$ there exists a unique sequence of scalars $\left(a_{i}\right)_{i=1}^{\infty}$ so that $x=\sum_{i=1}^{\infty} a_{i} x_{i}$ and the convergence is unconditional, i.e., $x=\sum_{i=1}^{\infty} a_{\pi(i)} x_{\pi(i)}$ for all permutations $\pi$ of $\mathbb{N}$. This is equivalent to all $x_{i}$ 's $\neq 0$, $\left[\left(x_{i}\right)_{i \in \mathbb{N}}\right]=X$ and

$$
\sup \left\{\left\|\sum_{i=1}^{\infty} \varepsilon_{i} a_{i} x_{i}\right\|: \sum_{i=1}^{\infty} a_{i} x_{i} \in B_{X} \text { and } \varepsilon_{i}= \pm 1 \text { for all } i\right\}<\infty .
$$

Here $B_{X}$ denotes the closed unit ball of $X$. This number is called the unconditional basis constant of $\left(x_{i}\right)_{i=1}^{\infty}$. The biorthogonal functionals then form an unconditional basic sequence in $X^{*}$.

A block basis $\left(y_{i}\right)_{i=1}^{\infty}$ of a basic sequence $\left(x_{i}\right)_{i=1}^{\infty}$ is a non-zero sequence given by

$$
y_{i}=\sum_{j=n_{i-1}+1}^{n_{i}} a_{j} x_{j} \text { for some sequence } n_{0}<n_{1}<n_{2}<\cdots
$$

in $\mathbb{N}_{0}$ and scalars $\left(a_{j}\right)_{j=1}^{\infty} \subseteq \mathbb{R}$. A block basis is a basic sequence, which is unconditional basic if the $x_{i}$ 's are unconditional basic. A sequence $\left(x_{i}\right)$ is semi-normalized if $0<\inf \left\|x_{i}\right\| \leq \sup _{i}\left\|x_{i}\right\|<\infty$.

A Schauder frame for a Banach space $X$ is a sequence $\left(x_{i}, f_{i}\right) \subseteq X \times X^{*}$ such that for all $x \in X, x=\sum_{i=1}^{\infty} f_{i}(x) x_{i}$. Of course every basis for $X$ is a frame for $X$ and just as in the basis case, the uniform boundedness principle yields $\sup \left\{\left\|\sum_{1}^{n} f_{i}(x) x_{i}\right\|: n \in \mathbb{N}, x \in S_{X}\right\}<\infty$ (called the frame constant) where $S_{X}=\{x \in X:\|x\|=1\}$ is the unit sphere of $X$. More on frames can be found in CHL and CDOSZ. Schauder frames should not be confused with Hilbert frames, which are much more restrictive. Note that for frames, $\left(x_{i}, f_{i}\right)$ is not assumed to be a biorthogonal sequence.

In our situation, where we are concerned with $\left(f_{i}\right)_{i=1}^{\infty}$ being a sequence of uniformly discrete translations of some $f \in L_{p}(\mathbb{R})$, we do not know of an example where $\left(f_{i}\right)$ is a frame but is not basic. However, many of our results would hold 
only given the property of Proposition 2.1 below and so we have stated them in terms of frames.

Some background material on $L_{p}$ spaces which we shall use can be found in [AOd] and in the basic concepts chapter of [JL. In particular we shall use that a normalized unconditional basic sequence $\left(f_{i}\right)$ in $L_{p}(\mathbb{R})$ satisfies for constants $A_{p}$ and $B_{p}$, depending on $p$ and the unconditional basis constant of $\left(f_{i}\right)$,

(1.1) For $1 \leq p \leq 2$, for all $\left(a_{i}\right) \subseteq \mathbb{R}$,

$$
A_{p}^{-1}\left(\sum_{i=1}^{\infty} a_{i}^{2}\right)^{1 / 2} \leq\left\|\sum_{i=1}^{\infty} a_{i} f_{i}\right\|_{p} \leq B_{p}\left(\sum_{i=1}^{\infty}\left|a_{i}\right|^{p}\right)^{1 / p} .
$$

(1.2) For $2<p<\infty,\left(a_{i}\right) \subseteq \mathbb{R}$,

$$
A_{p}^{-1}\left(\sum_{i=1}^{\infty}\left|a_{i}\right|^{p}\right)^{1 / p} \leq\left\|\sum_{i=1}^{\infty} a_{i} f_{i}\right\|_{p} \leq B_{p}\left(\sum_{i=1}^{\infty}\left|a_{i}\right|^{2}\right)^{1 / 2} .
$$

If $\left(f_{i}\right)$ is unconditional basic in $L_{p}[0,1], 1 \leq p<\infty$, then for some $C_{p}$, depending on $p$ and the unconditional basis constant of $\left(f_{i}\right)$, for all $\left(a_{i}\right) \subseteq \mathbb{R}$,

(1.3) (Square function inequality)

$$
\left\|\sum_{i=1}^{\infty} a_{i} f_{i}\right\|_{p} \stackrel{C_{p}}{\sim}\left(\int_{0}^{1}\left(\sum_{i=1}^{\infty}\left|a_{i}\right|^{2}\left|f_{i}(t)\right|^{2}\right)^{p / 2} d t\right)^{1 / p} .
$$

Here we use " $A \stackrel{C}{\sim} B$ " to denote $A \leq C B$ and $B \leq C A$.

The Haar basis $\left(h_{n}\right)_{n=1}^{\infty}$ is a basis for $L_{1}[0,1]$. This sequence is given by

$$
\begin{aligned}
\left(h_{n}\right)_{n=1}^{\infty}=\left(\mathcal{X}_{[0,1]},\right. & \mathcal{X}_{[0,1 / 2]}-\mathcal{X}_{[1 / 2,1]}, \\
\mathcal{X}_{[1 / 2,3 / 4]}-\mathcal{X}_{[3 / 4,1]}, & \left.\mathcal{X}_{[0,1 / 8]}-\mathcal{X}_{[1 / 8,1 / 4]}, \ldots\right) .
\end{aligned}
$$

The same system is an unconditional basis for $L_{p}[0,1], 1<p<\infty$. Usually below, we will let $\left(h_{n}\right)_{n=1}^{\infty}$ refer to the normalized Haar basis, i.e., $\left(h_{n} /\left\|h_{n}\right\|_{p}\right)_{n=1}^{\infty}$. We can get an unconditional basis for $L_{p}(\mathbb{R})$ from this by copying $\left(h_{n}\right)_{n=1}^{\infty}$ onto each

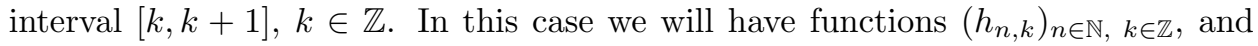
we will presume they are linearly ordered so as to be compatible with the Haar basis ordering on each $[k, k+1]$, i.e., if the functions are ordered as $\left(x_{i}\right)_{i=1}^{\infty}$ and if $x_{i}=h_{n, k}, x_{j}=h_{m, k}$ with $i<j$, then $n<m$. This ordering yields that if $\left(g_{i}\right)_{i=1}^{\infty}$ is a block basis of the Haar basis, then $\left(\left.g_{i}\right|_{[n, m]}\right)_{i=1}^{\infty}$ is also a block basis of the Haar basis (well, some $g_{i}$ 's could be 0 here) for all integers $n<m$.

The Rademacher sequence $\left(r_{n}\right)_{n=1}^{\infty}$ is given by $\left(r_{n}\right)_{n=1}^{\infty}=\left(h_{1}, h_{2}, h_{3}+h_{4}, h_{5}+\right.$ $\left.\cdots+h_{8}, \ldots\right)$, where the $h_{n}$ 's refer to the non-normalized Haar functions. The classical Khintchine's inequality asserts that it is equivalent to the unit vector basis of $\ell_{2}$ in all $L_{p}[0,1]$ spaces, $1 \leq p<\infty$, i.e.,

$$
\left\|\sum_{i=1}^{\infty} a_{i} r_{i}\right\|_{p} \stackrel{K_{p}}{\sim}\left(\sum_{i=1}^{\infty}\left|a_{i}\right|^{2}\right)^{1 / 2}
$$

One reason for taking $\Lambda$ to be uniformly discrete in our considerations is, as mentioned above, given by the easy

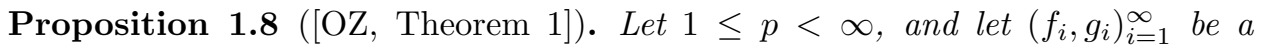
bounded biorthogonal system in $L_{p}(\mathbb{R})$ so that for some $f \in L_{p}(\mathbb{R})$ and $\left(\lambda_{i}\right)_{1}^{\infty} \subseteq \mathbb{R}$, $f_{i}=f_{\left(\lambda_{i}\right)}$ for all $i$. Then $\Lambda=\left(\lambda_{i}\right)_{1}^{\infty}$ is uniformly discrete. 
Proof. If not, there exist subsequences $\left(i_{m}\right)$ and $\left(j_{m}\right)$ of $\mathbb{N}$ so that

$$
\lim _{m \rightarrow \infty}\left|\lambda_{i_{m}}-\lambda_{j_{m}}\right|=0
$$

and $\lambda_{i_{m}} \neq \lambda_{j_{m}}$ for all $m$. Then

$$
\left\|g_{i_{m}}\right\| \geq \frac{\left\langle g_{i_{m}}, f_{i_{m}}-f_{j_{m}}\right\rangle}{\left\|f_{i_{m}}-f_{j_{m}}\right\|_{p}}=\frac{1}{\left\|f_{i_{m}}-f_{j_{m}}\right\|_{p}},
$$

and the latter is unbounded in $m$, a contradiction.

\section{MAin Results}

We begin with the elementary but very useful

Proposition 2.1. Let $\Lambda \subseteq \mathbb{R}$ be uniformly discrete, $1 \leq p<\infty$, and $f \in L_{p}(\mathbb{R})$. Then for all intervals $I=[a, b], \sum_{\lambda \in \Lambda}\left\|\left.f_{(\lambda)}\right|_{I}\right\|_{p}^{p}<\infty$.

Proof. Choose $\varepsilon_{0}>0$ so that $\left|\lambda-\lambda^{\prime}\right|>\varepsilon_{0}$ for all $\lambda, \lambda^{\prime} \in \Lambda$ with $\lambda \neq \lambda^{\prime}$. For $\ell \in \mathbb{Z}$, set $I_{\ell}=\left[a+(\ell-1) \varepsilon_{0}, a+\ell \varepsilon_{0}\right]$. Then for $\ell \in \mathbb{Z}$,

$$
\sum_{\lambda \in \Lambda}\left\|\left.f_{(\lambda)}\right|_{I_{\ell}}\right\|_{p}^{p}=\sum_{\lambda \in \Lambda} \int_{I_{\ell}}|f(t-\lambda)|^{p} d t=\sum_{\lambda \in \Lambda} \int_{a+(\ell-1) \varepsilon_{0}-\lambda}^{a+\ell \varepsilon_{0}-\lambda}|f(t)|^{p} d t \leq\|f\|_{p}^{p},
$$

since the intervals of integration are disjoint for $\lambda \in \Lambda$. Thus

$$
\sum_{\lambda \in \Lambda}\left\|\left.f_{(\lambda)}\right|_{I}\right\|_{p}^{p} \leq \sum_{\ell=1}^{\left\lceil\frac{b-a}{\varepsilon_{0}}\right\rceil} \sum_{\lambda \in \Lambda}\left\|\left.f_{(\lambda)}\right|_{I_{\ell}}\right\|_{p}^{p} \leq\left\lceil\frac{b-a}{\varepsilon_{0}}\right\rceil\|f\|_{p}^{p} .
$$

We note a simple consequence of Proposition 2.1. We remark that in AO, Theorem 4.1], it is proved that if $1<p<\infty$ and $f \in L_{p}(\mathbb{R}) \cap L_{1}(\mathbb{R})$, then $X_{p}(f, \mathbb{Z}) \neq L_{p}(\mathbb{R})$.

Proposition 2.2. Let $1<p<\infty, f \in L_{p}(\mathbb{R}) \cap L_{1}(\mathbb{R})$, and let $\left(f_{i}\right)_{i=1}^{\infty}$ be a sequence of uniformly discrete translates of $f$. Then $\left(f_{i}\right)_{i=1}^{\infty}$ is not a fundamental bounded minimal system for $L_{p}(\mathbb{R})$. Furthermore, there is no sequence $\left(g_{i}\right)_{i=1}^{\infty} \subseteq L_{q}(\mathbb{R})$ $(1 / p+1 / q=1)$ so that $\left(f_{i}, g_{i}\right)_{i=1}^{\infty}$ is a frame for $L_{p}(\mathbb{R})$.

Proof. Assume $\left(f_{i}, g_{i}\right)$ are in fact such a frame. $\left\|f_{i}\right\|_{p}=\|f\|_{p}$ for all $i$ and thus $\left(g_{i}\right)_{i=1}^{\infty}$ is $\omega^{*}$-null and hence bounded in $L_{q}(\mathbb{R})$. Let $K=\sup _{i}\left\|g_{i}\right\|_{q}$. Choose $n_{0} \in \mathbb{N}$ with

$$
\sum_{j=n_{0}+1}^{\infty}\left\|\left.f_{i}\right|_{[0,1]}\right\|_{1}<\frac{1}{4 K}
$$

Choose $h: \mathbb{R} \rightarrow \mathbb{R}$ so that $|h|=\chi_{[0,1]}$ and $\left|\left\langle h, g_{i}\right\rangle\right|<\frac{1}{4 n_{0}\|f\|_{1}}$ for $i \leq n_{0}(h$ could be a Rademacher function). Thus $\|h\|_{p}=\|h\|_{1}=1$. Also $h=\sum_{i=1}^{\infty}\left\langle h, g_{i}\right\rangle f_{i}$, the series converging in $L_{p}(\mathbb{R})$, and so

$$
\left.h\right|_{[0,1]}=\left.\sum_{i=1}^{\infty}\left\langle h, g_{i}\right\rangle f_{i}\right|_{[0,1]},
$$


the series converging in $L_{1}[0,1]$. Then

$$
\begin{aligned}
1 & =\|h\|_{1} \leq \sum_{i=1}^{\infty}\left|\left\langle h, g_{i}\right\rangle\right|\left\|\left.f_{i}\right|_{[0,1]}\right\|_{1} \\
& \leq \sum_{i=1}^{n_{0}}\left|\left\langle h, g_{i}\right\rangle\right|\|f\|_{1}+\sum_{i=n_{0}+1}^{\infty}\left\|g_{i}\right\|_{q}\left\|\left.f_{i}\right|_{[0,1]}\right\|_{1} \\
& <\frac{n_{0}}{4 n_{0}\|f\|_{1}}\|f\|_{1}+\sup _{i}\left\|g_{i}\right\|_{q} \frac{1}{4 K}=\frac{1}{2},
\end{aligned}
$$

a contradiction.

The argument is similar if we assume that $\left(f_{i}, g_{i}\right)_{i=1}^{\infty}$ is a fundamental bounded biorthogonal system for $L_{p}(\mathbb{R})$. Then, for the same $h, n_{0}$ and for $\varepsilon>0$ arbitrary, we can choose $f=\sum_{i=1}^{n} a_{i} f_{i}$ with $\|h-f\|_{p}<\varepsilon$. Thus $\left\|\left.f\right|_{[0,1]}-h\right\|_{1}<\varepsilon$ and

$$
1-\varepsilon \leq\left\|\left.f\right|_{[0,1]}\right\|_{1} \leq \sum_{i=1}^{n_{0}}\left|a_{i}\right|\left\|\left.f_{i}\right|_{[0,1]}\right\|_{1}+\sum_{i=n_{0}+1}^{n}\left|a_{i}\right|\left\|\left.f_{i}\right|_{[0,1]}\right\|_{1} .
$$

For $i \leq n_{0}$,

$$
\left|a_{i}\right|=\left|g_{i}(f)\right| \leq\left|g_{i}(f-h)\right|+\left|g_{i}(h)\right|<K \varepsilon+\frac{1}{4 n_{0}\|f\|_{1}} .
$$

For $i>n_{0},\left|a_{i}\right| \leq K(1+\varepsilon)$. Hence by (2.1)

$$
\begin{aligned}
& 1-\varepsilon \leq n_{0}\left(K \varepsilon+\frac{1}{4 n_{0}\|f\|_{1}}\right)\|f\|_{1}+\sum_{i=n_{0}+1}^{n} K(1+\varepsilon)\left\|\left.f_{i}\right|_{[0,1]}\right\|_{1} \\
&<n_{0} K \varepsilon\|f\|_{1}+\frac{1}{4}+\frac{1}{4}(1+\varepsilon)<\frac{3}{4}<1-\varepsilon
\end{aligned}
$$

a contradiction, if $\varepsilon<1 / 4$.

For $p=1$ we have a stronger result (Corollary 2.4) as a consequence of our next theorem.

Definition. Let $1 \leq p<\infty, 1 / p+1 / q=1$.

a) Let $\left(f_{i}, g_{i}\right) \subseteq L_{p}(\mathbb{R}) \times L_{q}(\mathbb{R})$ be a frame for a subspace $X$ of $L_{p}(\mathbb{R})$. We say $\left(f_{i}, g_{i}\right)$ satisfies $(*)$ if

(*) for all $\varepsilon>0$ and all bounded intervals $I \subseteq \mathbb{R}$, there exists $n \in \mathbb{N}$ so that for all $m \geq n$ and $f \in X$,

$$
\left\|\left.\sum_{i=m+1}^{\infty}\left\langle f, g_{i}\right\rangle f_{i}\right|_{I}\right\|_{p} \leq \varepsilon\|f\|_{p}
$$

b) A semi-normalized bounded minimal system $\left(f_{i}\right)_{i=1}^{\infty}$ in $L_{p}(\mathbb{R})$ satisfies $(* *)$ if

$(* *)$ for all $\varepsilon>0$ and bounded intervals $I \subseteq \mathbb{R}$ there exists $n \in \mathbb{N}$ so that for all $n<m \leq m_{1} \leq m_{2}$ and $f=\sum_{i=1}^{m_{2}} a_{i} f_{i}$ with $\|f\|_{p}=1,\left\|\left.\sum_{i=m}^{m_{1}} a_{i} f_{i}\right|_{I}\right\| \leq$ $\varepsilon$.

Theorem 2.3. Let $\left(f_{i}, g_{i}\right)_{i=1}^{\infty}$ be a frame or a semi-normalized bounded fundamental minimal system for a subspace $X$ of $L_{p}(\mathbb{R}), 1 \leq p<\infty$, satisfying $(*)$ or $(* *)$, respectively. Then $X$ embeds almost isometrically into $\ell_{p}$. 
That $X$ embeds almost isometrically into $\ell_{p}$ means that for all $\varepsilon>0$ there exists $T: X \rightarrow \ell_{p}$ with $(1+\varepsilon)^{-1} \leq\|T f\| \leq 1+\varepsilon$ for all $f \in S_{X}$. The proof of Theorem 2.3 will yield, for all $\varepsilon>0$, a partition $\Pi=\left(D_{s}\right)_{s=1}^{\infty}$ of $\mathbb{R}$ into intervals so that for all $f \in S_{X}$,

$$
\left\|f-\mathbb{E}_{\Pi} f\right\|_{p}<\varepsilon .
$$

$\mathbb{E}_{\Pi}$ is the conditional expectation projection

$$
f \longmapsto \sum_{s=1}^{\infty}\left(\int_{D_{s}} f\right) \frac{\chi_{D_{s}}}{m\left(D_{s}\right)}
$$

Of course, in $L_{p},\left(\frac{\chi_{D_{s}}}{m\left(D_{s}\right)}\right)$ is 1-equivalent to the unit vector basis of $\ell_{p}$.

From Proposition 2.1 and Theorem 2.3 we obtain

Corollary 2.4. If $\left(f_{i}, g_{i}\right)$ is a frame or a fundamental bounded minimal system for a subspace $X$ of $L_{1}(\mathbb{R})$ where the $f_{i}$ 's are uniformly discrete translates of some $f \in L_{1}(\mathbb{R})$, then $X$ embeds almost isometrically into $\ell_{1}$.

Proof of Theorem 2.3. We first consider the frame case and let $C$ be the frame constant. Thus for all $f \in X$ and $n \in \mathbb{N}$,

$$
\left\|\sum_{i=1}^{n}\left\langle f, g_{i}\right\rangle f_{i}\right\|_{p} \leq C\|f\|_{p}
$$

Let $\varepsilon>0$. We inductively choose increasing sequences $\left(m_{k}\right)$ and $\left(n_{k}\right)$ in $\mathbb{N}$ to obtain, where $I_{k}=\left[-m_{k}, m_{k}\right]$,

$$
\begin{aligned}
& \text { (2.2) for } f \in X, \text { and } n \geq n_{k}, \quad\left\|\left.\sum_{i=n+1}^{\infty}\left\langle g_{i}, f\right\rangle f_{i}\right|_{I_{k-1}}\right\|_{p} \leq \varepsilon 2^{-k}\|f\|_{p}, \\
& \text { (2.3) for } f \in \operatorname{span}\left\{f_{i}: i \leq n_{k}\right\}, \quad\left\|\left.f\right|_{\mathbb{R} \backslash I_{k}}\right\|_{p} \leq \varepsilon 2^{-k}\|f\|_{p} .
\end{aligned}
$$

We do this by setting $I_{0}=\emptyset$, letting $n_{1}$ be arbitrary, and choosing $m_{1}$ to satisfy (2.3) for $k=1$. Then we choose $n_{2}$ to satisfy (2.2) using $(*)$ and continue in this manner. We let $A_{k}=I_{k} \backslash I_{k-1}$, for $k \in \mathbb{N}$.

Choose a partition $\pi_{k}$ of $A_{k}$ into intervals, $k \geq 1$, so that for all $f \in$ $\operatorname{span}\left\{f_{i}: i \leq n_{k+1}\right\}$,

$$
\left\|\left.f\right|_{A_{k}}-\sum_{D \in \pi_{k}} \frac{\chi_{D}}{m(D)} \int_{D} f(x) d x\right\|_{p} \leq \varepsilon 2^{-k}\|f\|_{p} .
$$

Let $f \in X$ with $\|f\|_{p}=1$. Then, with $n_{0}=0$,

$$
\begin{aligned}
1 & =\left\|\sum_{i=1}^{\infty}\left\langle g_{i}, f\right\rangle f_{i}\right\|_{p}=\left\|\sum_{s=1}^{\infty}\left(\left.\sum_{i=n_{s-1}+1}^{n_{s}}\left\langle g_{i}, f\right\rangle f_{i}\right|_{I_{s}}\right)+\sum_{s=1}^{\infty}\left(\left.\sum_{i=n_{s-1}+1}^{n_{s}}\left\langle g_{i}, f\right\rangle f_{i}\right|_{\mathbb{R} \backslash I_{s}}\right)\right\|_{p} \\
& \leq\left\|\left.\sum_{s=1}^{\infty} \sum_{i=n_{s-1}+1}^{n_{s}}\left\langle g_{i}, f\right\rangle f_{i}\right|_{I_{s}}\right\|_{p}+2 C \varepsilon, \text { by (2.3) } \\
& \leq\left\|\left.\sum_{s=1}^{\infty} \sum_{i=n_{s-1}+1}^{n_{s}}\left\langle g_{i}, f\right\rangle f_{i}\right|_{I_{s} \backslash I_{s-2}}\right\|_{p}+2 C \varepsilon+2 \varepsilon, \text { by (2.2) }
\end{aligned}
$$


where we let $I_{-1}=I_{0}=\emptyset$

$$
=\left\|\left.\sum_{s=1}^{\infty} \sum_{i=n_{s-1}+1}^{n_{s}}\left\langle g_{i}, f\right\rangle f_{i}\right|_{A_{s} \cup A_{s-1}}\right\|_{p}+2 C \varepsilon+2 \varepsilon
$$

where we let $A_{0}=\emptyset$

$$
\begin{aligned}
& =\left\|\sum_{s=1}^{\infty} \chi_{A_{s}} \sum_{i=n_{s-1}+1}^{n_{s+1}}\left\langle g_{i}, f\right\rangle f_{i}\right\|_{p}+2 C \varepsilon+2 \varepsilon \\
& \leq\left(\sum_{s=1}^{\infty} \sum_{D \in \pi_{s}}\left|\int_{D} \sum_{i=n_{s-1}+1}^{n_{s+1}}\left\langle g_{i}, f\right\rangle f_{i}(x) d x\right|^{p}\right)^{1 / p}+4 C \varepsilon+2 \varepsilon \text { by (2.4) } .
\end{aligned}
$$

Now by (2.2) for $s \in \mathbb{N}$,

$$
\left(\sum_{D \in \pi_{s}}\left|\int_{D} \sum_{i=n_{s+1}+1}^{\infty}\left\langle g_{i}, f\right\rangle f_{i}(x) d x\right|^{p}\right)^{1 / p} \leq\left\|\left.\sum_{i=n_{s+1}+1}^{\infty}\left\langle g_{i}, f\right\rangle f_{i}\right|_{I_{s}}\right\|_{p} \leq \varepsilon 2^{-(s+1)} .
$$

If $s>1$, then by (2.3),

$$
\left(\sum_{D \in \pi_{s}}\left|\int_{D} \sum_{i=1}^{n_{s-1}}\left\langle g_{i}, f\right\rangle f_{i}(x) d x\right|^{p}\right)^{1 / p} \leq 2^{-s+1} C \varepsilon
$$

From (2.5), (2.6), and (2.7), we obtain that

$$
\begin{aligned}
1=\|f\|_{p} & \leq\left(\sum_{D \in \bigcup_{s=1}^{\infty} \pi_{s}}\left|\int_{D} f(x) d x\right|^{p}\right)^{1 / p}+\sum_{s=1}^{\infty} \varepsilon 2^{-(s+1)}+\sum_{s=2}^{\infty} 2^{-s+1} C \varepsilon+6 C \varepsilon \\
& \leq\left(\sum_{D \in \bigcup_{s=1}^{\infty} \pi_{s}}\left|\int_{D} f(x) d x\right|^{p}\right)^{1 / p}+8 C \varepsilon=1+8 C \varepsilon .
\end{aligned}
$$

Thus $T: X \rightarrow \ell_{p}\left(\bigcup_{s=1}^{\infty} \pi_{s}\right)$ given by $f \mapsto\left(\int_{D} f(x) d x\right)_{D \in \bigcup_{s=1}^{\infty} \pi_{s}}$ is the desired embedding.

The proof in the case that $\left(f_{i}\right)_{i=1}^{\infty}$ is a bounded fundamental minimal system for $X \subseteq L_{p}(\mathbb{R})$ is nearly identical. We let $K=\sup _{i}\left\|g_{i}\right\|_{q}$ and in the construction 
replace (2.2)-(2.4) by

$$
\begin{aligned}
& \text { For all } n_{k}<n \leq m \leq \bar{m} \text { and } f=\sum_{1}^{\bar{m}} a_{i} f_{i} \in S_{X}, \\
& \left\|\left.\sum_{i=n}^{m} a_{i} f_{i}\right|_{I_{k-1}}\right\|_{p} \leq \varepsilon 2^{-k} \quad(\operatorname{using}(* *)) .
\end{aligned}
$$

For all $f=\sum_{i=1}^{n_{k}} a_{i} f_{i}$ with $\left|a_{i}\right| \leq K$ for $i \leq n_{k}, \quad\left\|\left.f\right|_{\mathbb{R} \backslash I_{k}}\right\|_{p} \leq \varepsilon 2^{-k}$.

$$
\begin{gathered}
\text { For all } f=\sum_{i=1}^{n_{k+1}} a_{i} f_{i} \text { with }\left|a_{i}\right| \leq K \text { for } i \leq n_{k+1}, \\
\left\|\left.f\right|_{A_{k}}-\sum_{D \in \Pi_{k}} \frac{\chi_{D}}{m(D)} \int_{D} f(x) d x\right\|_{p} \leq \varepsilon 2^{-k} .
\end{gathered}
$$

The proof then proceeds as in the frame case for $f \in \operatorname{span}\left(f_{i}\right), f=\sum_{i=1}^{\ell} a_{i} f_{i}$, $\|f\|_{p}=1$.

Remark 2.5. Let $X \subseteq L_{p}(\mathbb{R})$ be as in Theorem 2.3 with $1<p<\infty$. Then there is a shorter proof that yields $X \hookrightarrow \ell_{p}$. In fact in the bounded minimal system case, one can replace $(* *)$ by the weaker

(***) For all $\varepsilon>0$ and bounded intervals $I \subseteq \mathbb{R}$, there exists $n \in \mathbb{N}$ so that if $f \in \operatorname{span}\left(f_{i}\right)_{i \geq n}$ with $\|f\|_{p}=1$, then $\left\|\left.f\right|_{I}\right\|_{p}<\varepsilon$.

Indeed by $\left[\mathrm{KP},[\mathrm{J}]\right.$ and $\left[\mathrm{JO}\right.$, it suffices to prove that if $\left(x_{n}\right)$ is a normalized weakly null sequence in $X$, then some subsequence is 2-equivalent to the unit vector basis of $\ell_{p}$. Then, from $(*)$ or $(* * *)$, it is easy to find $\left(x_{n_{i}}\right)$ and intervals $I_{1} \subseteq I_{2} \subseteq$ ... so that $\left\|\left.x_{n_{i}}\right|_{I_{i} \backslash I_{i-1}}\right\|_{p}>1-\frac{\varepsilon}{2^{i}}$ for all $i$ and deduce the result.

We will say that a frame $\left(f_{i}, g_{i}\right)_{i=1}^{\infty}$ for $X$ satisfies a lower $\ell_{q}$-estimate if for some $K<\infty$ and all $x \in X$,

$$
\left(\sum_{i=1}^{\infty}\left|g_{i}(x)\right|^{q}\right)^{1 / q} \leq K\left\|\sum_{i=1}^{\infty} g_{i}(x) f_{i}\right\|=K\|x\| .
$$

A Hilbert frame, by definition, satisfies lower (and upper) $\ell_{2}$-estimates.

If $\left(x_{i}\right)_{i=1}^{\infty}$ is a fundamental bounded minimal system for $X$, we say that $\left(x_{i}\right)_{i=1}^{\infty}$ satisfies a lower $\ell_{q}$-estimate if for some $K$ and all scalars $\left(a_{i}\right)_{1}^{n}$

$$
\left(\sum_{i=1}^{n}\left|a_{i}\right|^{q}\right)^{1 / q} \leq K\left\|\sum_{i=1}^{n} a_{i} x_{i}\right\|
$$

Proposition 2.6. Let $1<p<\infty, 1 / p+1 / q=1$.

a) Assume $1<p \leq 2$, and let $\left(f_{i}\right)_{i=1}^{\infty}$ be a sequence of uniformly discrete translations of $f \in L_{p}(\mathbb{R})$. Let either $\left(f_{i}, g_{i}\right)_{i=1}^{\infty} \subseteq L_{p}(\mathbb{R}) \times L_{q}(\mathbb{R})$ be a frame or $\left(f_{i}\right)_{i=1}^{\infty}$ be a fundamental bounded minimal system for $X \subseteq L_{p}(\mathbb{R})$. If $\left(f_{i}\right)_{i=1}^{\infty}$ admits a lower $\ell_{q}$-estimate, then $X$ embeds almost isometrically into $\ell_{p}$.

b) Let $\left(f_{i}, g_{i}\right)_{i=1}^{\infty}$ be a frame for $L_{p}(\mathbb{R})$, where $\left(f_{i}\right)_{i=1}^{\infty}$ is a sequence of uniformly discrete translations of $f \in L_{p}(\mathbb{R})$. Then for all bounded measurable sets $B$ of positive measure, $\sum_{i=1}^{\infty}\left\|\left.g_{i}\right|_{B}\right\|_{1}^{q}=\infty$. 
Remark 2.7. The hypothesis in a) would be vacuous for $p>2$ since some subsequence of $\left(f_{i}\right)$ is equivalent to the unit vector basis of $\ell_{p}$.

Proof. First let $\left(f_{i}, g_{i}\right)_{i=1}^{\infty}$ be a frame for $X \subseteq L_{p}(\mathbb{R})$ as in a). Assume for all $f \in X$,

$$
\left(\sum_{i=1}^{\infty}\left|g_{i}(f)\right|^{q}\right)^{1 / q} \leq K\|f\|_{p}
$$

For any bounded interval $I \subseteq \mathbb{R}, f \in X$ and $n \in \mathbb{N}$,

$$
\begin{aligned}
\left\|\left.\sum_{i=n}^{\infty} g_{i}(f) f_{i}\right|_{I}\right\|_{p} & \leq \sum_{i=n}^{\infty}\left|g_{i}(f)\right|\left\|\left.f_{i}\right|_{I}\right\|_{p} \\
& \leq\left(\sum_{i=n}^{\infty}\left|g_{i}(f)\right|^{q}\right)^{1 / q}\left(\sum_{i=n}^{\infty}\left\|\left.f_{i}\right|_{I}\right\|_{p}^{p}\right)^{1 / p} \\
& \leq K\|f\|_{p}\left(\sum_{i=n}^{\infty}\left\|\left.f_{i}\right|_{I}\right\|_{p}^{p}\right)^{1 / p}
\end{aligned}
$$

From Proposition 2.1 we obtain that $(*)$ holds and so Theorem 2.3 applies.

Similarly, if $\left(f_{i}\right)$ is a fundamental bounded minimal system for $X$ and $f=$ $\sum_{1}^{\bar{m}} a_{i} f_{i}$ with $\|f\|_{p}=1$, we have

$$
\left\|\left.\sum_{n}^{m} a_{i} f_{i}\right|_{I}\right\|_{p} \leq K\left(\sum_{n}^{m}\left\|\left.f_{i}\right|_{I}\right\|_{p}\right)^{1 / p}
$$

and so, again, we have $(* *)$ and apply Theorem 2.3

b) Assume that for some $B$ of positive measure $\sum_{i=1}^{\infty}\left\|\left.g_{i}\right|_{B}\right\|_{1}^{q}<\infty$. Let $h \in$ $L_{\infty}(B),|h|=1$. So $h=\left.\sum_{i=1}^{\infty}\left\langle h, g_{i}\right\rangle f_{i}\right|_{B}$, the series converging in $L_{1}(B)$. Thus

$$
\begin{aligned}
m(B)=\|h\|_{1} & \leq \sum_{i=1}^{n}\left|\left\langle h, g_{i}\right\rangle\right|\left\|f_{i}\right\|_{1}+\sum_{i=n+1}^{\infty}\left|\left\langle h,\left.g_{i}\right|_{B}\right\rangle\right|\left\|\left.f_{i}\right|_{B}\right\|_{1} \\
& \leq \sum_{i=1}^{n}\left|\left\langle h, g_{i}\right\rangle\right|\left\|f_{i}\right\|_{1}+\left(\sum_{i=n+1}^{\infty}\left\|\left.g_{i}\right|_{B}\right\|_{1}^{q}\right)^{1 / q}\left(\sum_{i=n+1}^{\infty}\left\|\left.f_{i}\right|_{B}\right\|_{1}^{p}\right)^{1 / p} .
\end{aligned}
$$

Then, as in the proof of Proposition 2.2, we can choose $n$ so that the second term does not exceed $m(B) / 4$, and given this $n$, choose $h$ to make the first term also less than $m(B) / 4$. Thus $m(B)<\frac{1}{2} m(B)$, a contradiction.

Part a) of Proposition 2.6 yields a quantitative improvement of Theorem 1.6, If $\left\{f_{(\lambda)}: \lambda \in \Lambda\right\}$ is unconditional basic in $L_{2}(\mathbb{R})$, then given $\varepsilon>0$ there is a partition $\Pi$ of $\mathbb{R}$ so that for all $g \in X_{2}(f, \Lambda)$,

$$
\left\|g-\mathbb{E}_{\Pi} g\right\|_{2} \leq \varepsilon\|g\|_{2} .
$$

Remark 2.8. a) Let $f=\chi_{[0,1]}-\chi_{[1,2]} \in L_{p}(\mathbb{R})$. The sequence $\left(f_{(n)}\right)_{n \in \mathbb{Z}}$ is basic in $L_{1}(\mathbb{R})$ when ordered as $\left(f_{(0)}, f_{(1)}, f_{(-1)}, f_{(2)}, f_{(-2)}, \ldots\right)$. It is not unconditional since in any $L_{p}(\mathbb{R})$

$$
\left\|\sum_{i=-2 n}^{2 n} f_{(i)}\right\|_{p}=2^{1 / p} \text { but }\left\|\sum_{i=-n}^{n} f_{(2 i)}\right\|_{p}=(2 n+2)^{1 / p} .
$$


For $1<p<\infty,\left(f_{(n)}\right)_{n \in \mathbb{Z}}$ is neither a frame nor a minimal system in $L_{p}(\mathbb{R})$. The latter follows easily from the fact that

$$
\lim _{n \rightarrow \infty}\left\|f_{(0)}+\sum_{k=1}^{n} \frac{n-k}{n}\left(f_{(k)}+f_{(-k)}\right)\right\|_{p}=0 .
$$

b) (Due to S.J. Dilworth.) Let $1 \leq p<\infty$, and let

$$
f=\chi_{[-3 / 2,-1 / 2]}+2 \chi_{[-1 / 2,1 / 2]}+\chi_{[1 / 2,3 / 2]} \text {. }
$$

For $n \in \mathbb{N}$, set

$$
g_{n}=f+\sum_{k=1}^{n}(-1)^{n} \frac{n-k+1}{n}\left(f_{(k)}+f_{(-k)}\right) .
$$

Then for $x \geq 0$,

$$
g_{n}(x)=\left\{\begin{array}{cl}
-f_{(-1)}(x)+f_{(0)}(x)-f_{(1)}(x)=-1+2-1=0 & \text { if } x \in\left[0, \frac{1}{2}\right], \\
f_{(0)}\left(x-f_{(1)}(x)+\frac{n-1}{n} f_{(2)}(x)=1-2+\frac{n-1}{n}=-\frac{1}{n}\right. & \text { if } x \in\left[\frac{1}{2}, \frac{3}{2}\right], \\
(-1)^{k} \frac{n-k+1}{n} f_{(k)}(x)+(-1)^{k+1} \frac{n-k}{n} f_{(k+1)}(x) & \\
+(-1)^{k+2} \frac{n-k-1}{n} f_{(k+2)}(x)=0 & \text { if } x \in\left[k+\frac{1}{2}, k+\frac{3}{2}\right] \\
(-1)^{n-1} \frac{2}{n} f_{(n-1)}(x)+(-1)^{n} \frac{1}{n} f_{(n)}(x)=0 & \text { for some } 1 \leq k \leq n-2, \\
(-1)^{n} \frac{1}{n} f_{(n)}(x)=(-1)^{n} \frac{1}{n} & \text { if } x \in\left[n-\frac{1}{2}, n+\frac{1}{2}\right],
\end{array}\right.
$$

Thus $\left\|g_{n}\right\|_{p}=4^{1 / p} / n$, hence $f_{(0)} \in\left[\left\{f_{(k)}: k \in \mathbb{Z} \backslash\{0\}\right\}\right]$ and so $\left(f_{(k)}\right)_{k \in \mathbb{Z}}$ is not a minimal system in $L_{p}(\mathbb{R})$. Furthermore,

$$
\hat{\chi}_{[-1 / 2,1 / 2]}(x)=\frac{1}{\sqrt{2 \pi}} \int_{-1 / 2}^{1 / 2} e^{-i x t} d t=\frac{1}{\sqrt{2 \pi}} \frac{\sin x}{x} .
$$

It follows that

$$
\hat{f}(x)=\frac{1}{\sqrt{2 \pi}} \frac{\sin x}{x}[2+\cos x]
$$

so $\hat{f}(x) \neq 0$ a.e.

c) (Due to D. Freeman.) It is well known that if $\left(f_{i}\right)_{i=1}^{\infty}$ is a normalized sequence in $L_{1}(\mathbb{R})$ with $\left\|\left.f_{i}\right|_{I_{i}}\right\| \geq \lambda>\frac{1}{2}$ for all $i$ and some sequence of pairwise disjoint measurable sets $I_{i} \subseteq \mathbb{R}$, then $\left(f_{i}\right)_{i=1}^{\infty}$ is equivalent to the unit vector basis of $\ell_{1}$. Indeed

$$
\begin{aligned}
\left\|\sum a_{i} f_{i}\right\|_{1} & \geq\left\|\left.\sum a_{i} f_{i}\right|_{I_{i}}\right\|_{1}-\sum\left|a_{i}\right|\left\|\left.f_{i}\right|_{\mathbb{R}_{i} \backslash I_{i}}\right\|_{1} \\
& \geq \lambda \sum\left|a_{i}\right|-(1-\lambda) \sum\left|a_{i}\right|=(2 \lambda-1) \sum\left|a_{i}\right| .
\end{aligned}
$$

Thus if $\left\{f_{(\lambda)}: \lambda \in \Lambda\right\}$ is a sequence of uniformly discrete translations of $0 \neq f \in$ $L_{1}(\mathbb{R})$, then it can be split into a finite number of subsequences, each equivalent to the unit vector basis of $\ell_{1}$.

d) By Theorem 1.4, if $1 \leq p<\infty$ and $f \in L_{p}(\mathbb{R}), f \neq 0$, then $\left\{f_{(\lambda)}: \lambda \in \mathbb{R}\right\}$ is linearly independent (see also Theorem 4.2 below). However, one can find $f \in$ $L_{1}(\mathbb{R})$ so that $\left\{f_{(n)}: n \in \mathbb{Z}\right\}$ is not $\omega$-linearly independent in its natural order $[\mathbb{R}$. 
We next turn to the case where $\left(f_{i}\right)$ is unconditional basic in $L_{p}$. We first recall

Proposition 2.9 ([JO, Lemma 2]). Let $1 \leq p \leq 2$. Let $\left(f_{i}\right) \subseteq L_{p}(\mathbb{R})$ be seminormalized and unconditional basic. Assume that for some $\delta>0$ there exists a sequence of disjoint measurable sets $\left(B_{i}\right)_{i=1}^{\infty}$ with $\left\|\left.f_{i}\right|_{B_{i}}\right\|_{p} \geq \delta$, for all $i$. Then $\left(f_{i}\right)_{i=1}^{\infty}$ is equivalent to the unit vector basis of $\ell_{p}$.

Corollary 2.10. Let $\left(f_{i}\right)_{i=1}^{\infty}$ be an unconditional basic sequence in $L_{p}(\mathbb{R}), 1 \leq p \leq$ 2. Assume the $f_{i}$ 's are all translates of some fixed $f \in L_{p}(\mathbb{R})$. Then $\left(f_{i}\right)_{i=1}^{\infty}$ is equivalent to the unit vector basis of $\ell_{p}$.

Proof. Let $f_{i}=f_{\left(\lambda_{i}\right)}$ for $i \in \mathbb{N}$. Let $\rho \equiv \frac{1}{2} \inf \left\{\left|\lambda_{i}-\lambda_{j}\right|: i \neq j\right\}>0$. Let $I$ be an interval of length $\rho$ with $\left\|\left.f\right|_{I}\right\|_{p}=\delta>0$. If $B_{i}=I+\lambda_{i}$ for $i \in \mathbb{N}$, then the $B_{i}$ 's are pairwise disjoint and $\left\|\left.f_{i}\right|_{B_{i}}\right\|=\left\|\left.f\right|_{I}\right\|=\delta$ for all $i$. Proposition 2.9 yields the result.

As we shall see, the situation is more complicated for $p>2$, and it is especially so for $p>4$.

Theorem 2.11. Let $2<p \leq 4$, and let $\left(f_{i}\right) \subseteq L_{p}(\mathbb{R})$ be an unconditional basis for $X \subseteq L_{p}(\mathbb{R})$. Assume the $f_{i}$ 's are all translates of some fixed $f \in L_{p}(\mathbb{R})$. Then $X$ embeds isomorphically into $\ell_{p}$.

Lemma 2.12. Let $p \neq 2$, and let $X$ be a subspace of $L_{p}(\mathbb{R})$ not containing an isomorph of $\ell_{p}$. Then there exists $c>0$ so that $\|f\|=\left\|\left.f\right|_{[-c, c]}\right\|_{p}$ is an equivalent norm on $X$.

Proof. If the lemma is false, then we can find $\left(f_{k}\right)_{k=1}^{\infty} \subseteq S_{X}$ and $\left(m_{k}\right)_{k=1}^{\infty} \subseteq \mathbb{N}$ so that $\left\|\left.f_{k}\right|_{\left[-m_{k}, m_{k}\right]}\right\| \geq 1-2^{-2 k-1}$ and $\left\|\left.f_{k+1}\right|_{\left[-m_{k}, m_{k}\right]}\right\| \leq 2^{-2 k-1}$ for all $k \in \mathbb{N}$. It follows easily that $\left(f_{k}\right)_{k=1}^{\infty}$ is equivalent to $\left(\left.f_{k}\right|_{\left[-m_{k}, m_{k}\right] \backslash\left[-m_{k-1}, m_{k-1}\right]}\right)_{k=1}^{\infty}$ which, being semi-normalized and disjointly supported, is equivalent to the unit vector basis of $\ell_{p}$.

We shall also use

Proposition 2.13 ([J]). Let $X$ be a subspace of $L_{p}(\mathbb{R}), 2<p<\infty$, which does not contain an isomorph of $\ell_{2}$. Then $X$ embeds isomorphically into $\ell_{p}$.

In fact by $\mathrm{KW}, X$ must then embed almost isometrically into $\ell_{p}$.

We set some notation and recall some things before proving the theorem. We let $\left(h_{i}\right)$ denote the normalized Haar basis for $L_{p}[0,1]$ regarded, canonically, as a subspace of $L_{p}(\mathbb{R})$. As mentioned in the introduction, for $i \in \mathbb{N}$ and $n \in \mathbb{Z}$, we let $h_{(i, n)}(\cdot)=h_{i}((\cdot)-n)$. Thus, $\left(h_{(i, n)}\right)_{i \in \mathbb{N}, n \in \mathbb{Z}}$ is an unconditional basis for $L_{p}(\mathbb{R})$.

G. Schechtman [S] made the very useful observation that if $\left(f_{i}\right)_{i=1}^{\infty}$ and $\left(g_{i}\right)_{i=1}^{\infty}$ are semi-normalized unconditional basic sequences in $L_{p}(\mathbb{R}), 1<p<\infty$, with

$$
\sum_{i=1}^{\infty}\left\|\left|f_{i}\right|-\left|g_{i}\right|\right\|_{p}<\infty
$$

then $\left(f_{i}\right)_{i=1}^{\infty}$ is equivalent to $\left(g_{i}\right)_{i=1}^{\infty}$. This follows from (1.3). In particular, if $\left(f_{i}\right)_{i=1}^{\infty}$ is semi-normalized unconditional basic in $L_{p}(\mathbb{R})$, then, by first approximating each $\left(f_{i}\right)_{i=1}^{\infty}$ by a simple dyadic function and then using the above consequence of (1.3), there exists a block basis $\left(g_{i}\right)_{i=1}^{\infty}$ of $\left(h_{(i, n)}\right)_{i \in \mathbb{N}, n \in \mathbb{Z}}$ satisfying (2.8) and thus being equivalent to $\left(f_{i}\right)_{i=1}^{\infty}$. 
Proof of Theorem 2.11. By Proposition 2.13, it suffices to prove that $X$ does not contain an isomorph of $\ell_{2}$. By our remarks above we can choose a block basis $\left(g_{i}\right)_{i=1}^{\infty}$ of $\left(h_{(i, n)}\right)$ which satisfies (2.8). In particular $\left(g_{i}\right)_{i=1}^{\infty}$ is equivalent to $\left(f_{i}\right)_{i=1}^{\infty}$, and we maintain

$$
\sum_{i=1}^{\infty}\left\|\left.g_{i}\right|_{I}\right\|_{p}^{p}<\infty \text { for all bounded intervals } I .
$$

Thus we need only show that $\left[\left(g_{i}\right)_{i=1}^{\infty}\right]$ does not contain an isomorph of $\ell_{2}$. If this is false, then there exists a normalized block basis $\left(\bar{g}_{i}\right)_{i=1}^{\infty}$ of $\left(g_{i}\right)_{i=1}^{\infty}$ which is equivalent to the unit vector basis of $\ell_{2}$. Set $\bar{X}=\left[\left(\bar{g}_{i}\right)_{i=1}^{\infty}\right]$. By Lemma 2.12 there exists $M \in \mathbb{N}$ and $1 \leq C<\infty$ so that for all $\bar{g} \in \bar{X}, I=[-M, M]$,

$$
\left\|\left.\bar{g}\right|_{I}\right\|_{p} \geq C^{-1}\|\bar{g}\|_{p} .
$$

Since $\left(g_{i}\right)_{i=1}^{\infty}$ is a block basis of $\left(h_{(i, n)}\right)$, then so is the normalized and unconditional sequence $\left(\left.g_{i}\right|_{I} /\left\|\left.g_{i}\right|_{I}\right\|_{p}\right)_{i=1}^{\infty}$. This yields lower $\ell_{p}$ and upper $\ell_{2}$ estimates for this sequence and $\left(g_{i}\right)$ (see (1.2)). From this and (2.10), we obtain for some constant $D<\infty$ and for all $\left(a_{i}\right) \subseteq \mathbb{R}$, and $\bar{g}=\sum_{i=1}^{\infty} a_{i} g_{i} \in \bar{X}$,

$$
\begin{aligned}
D^{-1}\left(\sum_{i=1}^{\infty}\left|a_{i}\right|^{p}\right)^{1 / p} & \leq D^{-1 / 2}\left\|\sum_{i=1}^{\infty} a_{i} g_{i}\right\|_{p} \\
& \leq\left\|\left.\sum_{i=1}^{\infty} a_{i} g_{i}\right|_{I}\right\|_{p}=\left.\left\|\sum_{i=1}^{\infty} a_{i}\right\| g_{i}\right|_{I}\left\|_{p} \frac{\left.g_{i}\right|_{I}}{\left\|\left.g_{i}\right|_{I}\right\|_{p}}\right\|_{p} \\
& \leq D\left(\sum_{i=1}^{\infty}\left|a_{i}\right|^{2}\left\|\left.g_{i}\right|_{I}\right\|_{p}^{2}\right)^{1 / 2} .
\end{aligned}
$$

By (2.9) there exists $n_{0} \in \mathbb{N}$ with

$$
\left(\sum_{i=n_{0}}^{\infty}\left\|\left.g_{i}\right|_{I}\right\|_{p}^{p}\right)^{1 / p}<(2 C)^{-1} D^{-2} .
$$

Let $\bar{g}$ be an element of $S_{X}$ which has the property that if we expand it in terms of the $g_{i}$ 's, i.e., if we write it as $\bar{g}=\sum_{i=1}^{\infty} a_{i} g_{i}$, then $a_{j}=0$ for $j \leq n_{0}$. From (2.10) and (2.11),

$$
\begin{aligned}
C^{-1} & \leq\left\|\left.\bar{g}\right|_{I}\right\|_{p} \leq D\left(\sum_{i=n_{0}}^{\infty} a_{i}^{2}\left\|\left.g_{i}\right|_{I}\right\|_{p}^{2}\right)^{1 / 2} \\
& \leq D\left[\left\|\left(a_{i}^{2}\right)_{i=n_{0}}^{\infty}\right\|_{\ell_{p / 2}} \cdot\left\|\left(\left\|\left.g_{i}\right|_{I}\right\|_{p}^{2}\right)_{i=n_{0}}^{\infty}\right\|_{\ell_{p} p}\right]^{1 / 2}
\end{aligned}
$$

(applying Hölder's inequality for $p / 2$ and $p / p-2$ )

$$
\begin{aligned}
& =D\left\|\left(a_{i}\right)_{i=n_{0}}^{\infty}\right\|_{\ell_{p}}\left(\sum_{i=n_{0}}^{\infty}\left\|\left.g_{i}\right|_{I}\right\|_{p}^{\frac{2 p}{p-2}}\right)^{\frac{p-2}{2 p}} \\
& \leq D^{2}\left\|\left.\bar{g}\right|_{I}\right\|_{p}\left(\sum_{i=n_{0}}^{\infty}\left\|\left.g_{i}\right|_{I}\right\|_{p}^{p}\right)^{1 / p}
\end{aligned}
$$


(by (2.11) and since $p \leq 4, \frac{2 p}{p-2} \geq p$ )

$$
\leq(2 C)^{-1} \text { by (2.12), }
$$

which is a contradiction.

When $p>4$ the possible structure is more complicated.

Theorem 2.14. Let $4<p<\infty$. There exists $f \in L_{p}(\mathbb{R})$ and $\Lambda \subseteq \mathbb{Z}$ so that $\left(f_{(\lambda)}\right)_{\lambda \in \Lambda}$ is an unconditional basic sequence with $X_{p}(f, \Lambda)$ containing an isomorph of $L_{p}(\mathbb{R})$.

Proof. We identify, in the canonical way, $L_{p}(\mathbb{R})$ with $\left(\bigoplus_{i \in \mathbb{Z}} L_{p}[0,1]\right)_{\ell_{p}}$. Since $L_{p}[0,1]$ is isometrically isomorphic to $L_{p}\left([0,1]^{2}\right)$, we need only produce $f=$ $\left(f_{i}\right)_{i \in \mathbb{Z}} \in\left(\bigoplus_{i \in \mathbb{Z}} L_{p}[0,1]^{2}\right)_{\ell_{p}}$ and $\Lambda \subseteq \mathbb{N}$ so that setting for $\lambda \in \Lambda f_{(\lambda)}=\left(f_{i-\lambda}\right)_{i \in \mathbb{Z}}$, then $X_{p}(f, \Lambda)$ contains an isomorph of $L_{p}[0,1]$ and $\left(f_{(\lambda)}\right)_{\lambda \in \Lambda}$ is unconditional.

Letting, as before, $\left(h_{n}\right)_{n=1}^{\infty}$ be the normalized Haar basis for $L_{p}[0,1]$ and $\left(r_{n}\right)_{n=1}^{\infty}$ the Rademacher functions on $[0,1]$, we have, for some constants $C_{p}$ and $D_{p}$ (see (1.3)) for all $\left(a_{i}\right) \subseteq \mathbb{R}$

$$
\left(\sum_{i=1}^{\infty}\left|a_{i}\right|^{2}\right)^{1 / 2} \leq\left\|\sum_{i=1}^{\infty} a_{i} r_{i}\right\|_{p} \leq C_{p}\left(\sum_{i=1}^{\infty}\left|a_{i}\right|^{2}\right)^{1 / 2}
$$

and

$$
\left\|\sum_{i=1}^{\infty} a_{i} h_{i}\right\|_{p} \stackrel{D_{p}}{\sim}\left\|\sum_{i=1}^{\infty} a_{i}^{2}\left|h_{i}\right|^{2}\right\|_{p / 2}^{1 / 2}
$$

Since $p>4$, we can choose $\left(\varepsilon_{i}\right)_{i=1}^{\infty} \subseteq(0,1)$ so that

$$
\sum_{i=1}^{\infty} \varepsilon_{i}^{p}=1
$$

and there exists a partition $\left(J_{n}\right)_{n=1}^{\infty}$ of $\mathbb{N}$ into finite intervals with

$$
\sum_{j \in J_{n}} \varepsilon_{j}^{4}=1 \text { for all } n \in \mathbb{N} .
$$

We are ready to define $f=\left(f_{i}\right)_{i \in \mathbb{Z}} \in\left(\bigoplus_{i \in \mathbb{Z}} L_{p}[0,1]^{2}\right)_{\ell_{p}}$. Set for $i \in \mathbb{Z}$,

$$
f_{i}=\left\{\begin{array}{l}
\varepsilon_{j} h_{n} \otimes r_{j}, \quad \text { if } i=3^{j} \text { with } j \in J_{n} \text { for some } n \in \mathbb{N}, \\
0, \text { otherwise }
\end{array}\right.
$$

where $h_{n} \otimes r_{j}$ is placed on the $i$ th copy of $[0,1]^{2}$. Note that

$$
\|f\|_{p}^{p}=\sum_{n \in \mathbb{N}} \sum_{j \in J_{n}}\left\|\varepsilon_{j} h_{n} \otimes r_{j}\right\|_{p}^{p}=\sum_{n \in \mathbb{N}} \sum_{j \in J_{n}} \varepsilon_{j}^{p}=1 .
$$

Let $\Lambda=\left\{-3^{j}: j \in \mathbb{N}\right\}$, and so our translated sequence is $\left(f_{\left(-3^{j}\right)}\right)_{j=1}^{\infty}$. For ease of notation below we shall write $f_{\left(-3^{j}\right)}, f$ shifted $3^{j}$ units left, as $f^{\left(-3^{j}\right)}$, and $f^{\left(-3^{j}\right)}=\left(f_{i}^{\left(-3^{j}\right)}\right)_{i \in \mathbb{Z}}$, where $f_{i}^{\left(-3^{j}\right)}$ denotes $f^{\left(-3^{j}\right)}$ restricted to the $i$ th $[0,1]^{2}$. 
Now $f_{0}^{\left(-3^{j}\right)}=\varepsilon_{j} h_{n} \otimes r_{j}$, if $j \in J_{n}$ and so for $\left(a_{j}\right) \subseteq \mathbb{R}$,

$$
\begin{aligned}
\left\|\sum_{j \in \mathbb{N}} a_{j} f_{0}^{\left(-3^{j}\right)}\right\|_{p}^{p} & =\left\|\sum_{n \in \mathbb{N}} \sum_{j \in J_{n}} a_{j} \varepsilon_{j} h_{n} \otimes r_{j}\right\|_{p}^{p} \\
& =\int_{0}^{1} \int_{0}^{1}\left|\sum_{n \in \mathbb{N}} \sum_{j \in J_{n}} a_{j} \varepsilon_{j} h_{n}(s) r_{j}(t)\right|^{p} d t d s \\
& \stackrel{C_{p}^{p}}{\sim} \int_{0}^{1}\left|\sum_{n \in \mathbb{N}} \sum_{j \in J_{n}} a_{j}^{2} \varepsilon_{j}^{2} h_{n}^{2}(s)\right|^{p / 2} d s, \text { by (2.13) } \\
& =\left\|\sum_{n \in \mathbb{N}}\left(\sum_{j \in J_{n}} a_{j}^{2} \varepsilon_{j}^{2}\right) h_{n}^{2}\right\|_{p / 2}^{p / 2} \\
& \sim D_{p}^{p}\left\|\sum_{n \in \mathbb{N}}\left(\sum_{j \in J_{n}} a_{j}^{2} \varepsilon_{j}^{2}\right)^{1 / 2} h_{n}\right\|_{p}^{p}, \text { by (2.14). }
\end{aligned}
$$

Now for $j \in \mathbb{N}, f_{\ell}^{\left(-3^{j}\right)} \neq 0$, iff $\ell=3^{k}-3^{j}$ for some $k \in \mathbb{N}$. If $\ell \neq 0$ and $\ell=3^{k}-3^{j}=3^{k^{\prime}}-3^{j^{\prime}}$ for $k, k^{\prime}, j, j^{\prime} \in \mathbb{N}$, then $k=k^{\prime}$ and $j=j^{\prime}$. Thus the functions $\left(f^{\left(-3^{j}\right)}\right)_{j \in \mathbb{N}}$ are disjointly supported except on the 0th copy of $[0,1]^{2}$. Also

$$
\left\|\sum_{\substack{\ell \neq 0 \\ \ell \in \mathbb{Z}}} f_{\ell}^{\left(-3^{j}\right)}\right\|_{p}^{p}=1-\varepsilon_{j}^{p}
$$

From this and (2.18) we obtain for some $K$, for all $\left(a_{i}\right) \subseteq \mathbb{R}$,

$$
\left\|\sum_{j \in \mathbb{N}} a_{j} f^{\left(-3^{j}\right)}\right\|_{p}^{p} \underset{\sim}{\sim}\left\|\sum_{n \in \mathbb{N}}\left(\sum_{j \in J_{n}} a_{j}^{2} \varepsilon_{j}^{2}\right)^{1 / 2} h_{n}\right\|_{p}^{p}+\sum_{j \in \mathbb{N}}\left|a_{j}\right|^{p} .
$$

Thus $\left(f^{\left(-3^{j}\right)}\right)_{j=1}^{\infty}$ is unconditional and we shall next construct a block basis $\left(b^{(n)}\right)_{n=1}^{\infty}$ of $\left(f^{\left(-3^{j}\right)}\right)_{j=1}^{\infty}$ which is equivalent to $\left(h_{n}\right)_{n=1}^{\infty}$.

For $n \in \mathbb{N}$, set

$$
b^{(n)}=\sum_{j \in J_{n}} \varepsilon_{j} f^{\left(-3^{j}\right)} .
$$

From (2.19), for $\left(c_{n}\right) \subseteq \mathbb{R}$

$$
\begin{aligned}
\left\|\sum_{n=1}^{\infty} c_{n} b^{(n)}\right\|_{p}^{p} & =\left\|\sum_{n=1}^{\infty} c_{n} \sum_{j \in J_{n}} \varepsilon_{j} f^{\left(-3^{j}\right)}\right\|_{p}^{p} \\
& \stackrel{K^{p}}{\sim}\left\|\sum_{n=1}^{\infty} c_{n}\left(\sum_{j \in J_{n}} \varepsilon_{j}^{4}\right)^{1 / 2} h_{n}\right\|_{p}^{p}+\sum_{n=1}^{\infty} \sum_{j \in J_{n}}\left|c_{n}\right|^{p} \varepsilon_{j}^{p} \\
& =\left\|\sum_{n=1}^{\infty} c_{n} h_{n}\right\|_{p}^{p}+\sum_{n=1}^{\infty}\left|c_{n}\right|^{p}\left(\sum_{j \in J_{n}} \varepsilon_{j}^{p}\right) .
\end{aligned}
$$

Thus, using this and (1.2), the lower $\ell_{p}$-estimate of $\left(h_{n}\right)_{n=1}^{\infty}$, we see that $\left(b^{(n)}\right)_{n=1}^{\infty}$ is equivalent to $\left(h_{n}\right)_{n=1}^{\infty}$. 
We next note that under certain additional assumptions, we cannot have the situation of Theorem 2.14,

Proposition 2.15. Let $4<p<\infty$, and let $\left(f_{i}\right)_{i=1}^{\infty}$ be an unconditional basis for $X \subseteq L_{p}(\mathbb{R})$ where the $f_{i}$ 's are all translations of some fixed $f \in L_{p}(\mathbb{R})$. If either

a) $f \in L_{2}(\mathbb{R})$ or

b) $\sum_{n \in \mathbb{Z}}\left\|\left.f\right|_{[n-1, n]}\right\|_{p}^{\frac{2 p}{p-2}}<\infty$,

then $X$ embeds isomorphically into $\ell_{p}$.

Proof. b) follows easily from the proof of Theorem 2.11. Indeed we can use b) to deduce the next to last inequality in that proof rather than using $p \leq 4$ as was done there.

a) We assume the contrary, so by Proposition $2.13 X$ contains an isomorph of $\ell_{2}$. We choose $I,\left(g_{i}\right)_{i=1}^{\infty}$ and $\left(\bar{g}_{i}\right)_{i=1}^{\infty}$ as in the proof of Theorem 2.11 with the additional assumption that $\sum_{i=1}^{\infty}\left\|\left|f_{i}\right|-\left|g_{i}\right|\right\|_{2}<\infty$.

Hence, using $f \in L_{2}(\mathbb{R})$,

$$
\sum_{i=1}^{\infty}\left\|\left.g_{i}\right|_{I}\right\|_{2}^{2}<\infty
$$

Now $\left(\left.\bar{g}_{i}\right|_{I}\right)_{i=1}^{\infty}$ is a block basis of $\left(h_{(i, n)}\right)$ which is equivalent to the unit vector basis of $\ell_{2}$. This forces $\|\cdot\|_{p}$ and $\|\cdot\|_{2}$ to be equivalent on $\left[\left(\left.\bar{g}_{i}\right|_{I}\right)_{i=1}^{\infty}\right] \subseteq L_{p}(I)$ KP. $\left(\bar{g}_{i}\right)_{i=1}^{\infty}$ is also a normalized block basis of $\left(g_{i}\right)_{i=1}^{\infty}$, and so we may write $\bar{g}_{i}=\sum_{j=n_{i-1}+1}^{n_{i}} c_{j} g_{j}$ for some scalars $\left(c_{j}\right), n_{0}<n_{1}<\cdots$ and all $i \in \mathbb{N}$. Since $\left(\left.g_{i}\right|_{I}\right)_{i=1}^{\infty}$ is also a block basis of $\left(h_{(i, n)}\right)$ and hence is orthogonal in $L_{2}(I)$, we have for $i \in \mathbb{N}$,

$$
\begin{aligned}
\left\|\left.\bar{g}_{i}\right|_{I}\right\|_{2} & =\left\|\left.\sum_{j=n_{i-1}+1}^{n_{i}} c_{j} g_{i}\right|_{I}\right\|_{2}=\left(\sum_{j=n_{i-1}+1}^{n_{i}} c_{j}^{2}\left\|\left.g_{j}\right|_{I}\right\|_{2}^{2}\right)^{1 / 2} \\
& \leq \sup _{j}\left|c_{j}\right|\left(\sum_{j=n_{i-1}+1}^{n_{i}}\left\|\left.g_{i}\right|_{I}\right\|_{2}^{2}\right)^{1 / 2}
\end{aligned}
$$

and the latter converges to 0 as $i \rightarrow \infty$ by (2.20). Thus $\left\|\left.\bar{g}_{i}\right|_{I}\right\|_{2} \rightarrow 0$ so $\left\|\left.\bar{g}_{i}\right|_{I}\right\|_{p} \rightarrow 0$ which is a contradiction.

We next present two more examples. The first is an easy example of a translation sequence in $L_{p}(2<p)$ which is unconditional but not equivalent to the $\ell_{p}$-basis and so Theorem 2.11 cannot be improved to get $\left(f_{i}\right)$ equivalent to the unit vector basis of $\ell_{p}$. The second is a translation sequence $\left(f_{i}\right)$ in $L_{p}, p>4$, which is basic but not unconditional.

Example 2.16. Let $2<p<\infty$. There exists $f \in L_{p}(\mathbb{R})$ so that $\left(f_{(n)}\right)_{n=1}^{\infty}$, the sequence of translations of $f$ by $n \in \mathbb{N}$, is unconditional basic but not equivalent to the unit vector basis of $\ell_{p}$.

Of course we already know this for $p>4$ by Theorem 2.14. Let $\left(r_{n}\right)_{n \in \mathbb{Z}}$ be an enumeration of the Rademacher functions on $[0,1]$ extended trivially to functions defined on all of $\mathbb{R}$. We define $\tilde{r}_{n}(\cdot)=r_{n}((\cdot)-n)$, for $n \in \mathbb{Z}$, and let $f=\sum_{n \in \mathbb{Z}} \frac{\tilde{r}_{n}}{\sqrt{|n|}}$, where we regard $\frac{1}{\sqrt{|0|}}=1$. Note that $\|f\|_{p}^{p}=1+2 \sum_{n=1}^{\infty} n^{-p / 2}<\infty$, since $p>2$. 
For $\left(a_{i}\right) \in c_{00}, g=\sum a_{i} f_{(i)}$ and $x \in[k, k+1], k \in \mathbb{Z}$, we observe

$$
g(x)=\sum_{i=1}^{\infty} a_{i} f(x-i)=\sum_{i=1}^{\infty} a_{i} \frac{r_{k-i}(x-k)}{\sqrt{|k-i|}} .
$$

Thus, for some $c_{p}>0$

$$
\|g\|_{p}^{p}=\sum_{k \in \mathbb{Z}}\left\|\left.g\right|_{[k, k+1]}\right\|_{p}^{p} \stackrel{c_{p}}{\sim} \sum_{k \in \mathbb{Z}}\left(\sum_{i=1}^{\infty} \frac{a_{i}^{2}}{|k-i|}\right)^{p / 2}
$$

which shows that $\left(f_{(i)}\right)_{i=1}^{\infty}$ is unconditional. Moreover, if we let $a_{i}=1$, for $i=$ $1, \ldots, m \in \mathbb{N}$ for $m \in \mathbb{N}$, we obtain

$$
\left\|\sum_{i=1}^{m} f_{(i)}\right\|_{p}^{p} \geq c_{p} \sum_{k=1}^{m}\left(\sum_{i=1}^{m} \frac{1}{|m-i|}\right)^{p / 2} \geq c_{p} m(\log m)^{p / 2} .
$$

Thus $\left(f_{(i)}\right)$ is not equivalent to the unit vector basis of $\ell_{p}$.

Example 2.17. Let $p>4$. There exists $f \in L_{p}(\mathbb{R})$ and $\Lambda \subset \mathbb{Z}$ so that $\left\{f_{(\lambda)}: \lambda \in\right.$ $\Lambda\}$ is basic in some order, but not unconditional.

As in Theorem 2.14 we identify $L_{p}(\mathbb{R})$ with $\left(\bigoplus_{n \in \mathbb{Z}} L_{p}[0,1]\right)_{p}$, and we write $f$ as $\left(f_{i}: \in \mathbb{Z}\right)$ with $f_{i} \in L_{p}(0,1)$, for $i \in \mathbb{Z}$, and, as in Theorem 2.14, we write $f^{(\lambda)}$ instead of $f_{(\lambda)}$.

For $j \in \mathbb{N}$, let $a_{j}=j^{-1 / 4}$ and $a_{0}=1$. Let $\left(r_{j}\right)$ be the Rademacher sequence on $[0,1]$. We define $f=\left(f_{i}\right)_{i \in \mathbb{Z}}$ by

$$
f_{i}= \begin{cases}a_{j-1} r_{j}-a_{j+1} r_{j+1}, & \text { if } i=3^{j} \text { for some } j \in \mathbb{N}, \\ 0, & \text { otherwise. }\end{cases}
$$

Since $p>4,\left(a_{i}\right) \in \ell_{p}$ and, thus, $f \in\left(\bigoplus_{n \in \mathbb{Z}} L_{p}[0,1]\right)_{p}$. We let $\Lambda=\left\{-3^{j}: j \in \mathbb{N}\right\}$. For $\left(b_{j}\right)_{j=1}^{n} \subset \mathbb{R}$ we compute $\left(b_{0}=0\right)$

$$
\begin{aligned}
\sum_{j=1}^{n} b_{j} f_{0}^{\left(-3^{j}\right)} & =\sum_{j=1}^{n} b_{j} f_{3^{j}} \\
& =\sum_{j=1}^{n} b_{j}\left(a_{j-1} r_{j}-a_{j+1} r_{j+1}\right) \\
& =\sum_{j=1}^{n}\left(b_{j} a_{j-1}-b_{j-1} a_{j}\right) r_{j}-b_{n} a_{n+1} r_{n+1} .
\end{aligned}
$$

We deduce that

$$
\begin{aligned}
\left\|\sum_{j=1}^{n} a_{j} f_{0}^{\left(-3^{j}\right)}\right\|_{p}=\| r_{1}- & a_{n} a_{n+1} r_{n+1} \|_{p} \rightarrow 1 \text { if } n \rightarrow \infty, \text { and } \\
\left\|\sum_{j=1}^{n}(-1)^{j+1} a_{j} f_{0}^{\left(-3^{j}\right)}\right\|_{p} & =\left\|r_{1}+\sum_{i=2}^{n}(-1)^{i+1} 2 a_{i-1} a_{i} r_{i} \pm a_{n} a_{n+1} r_{n+1}\right\|_{p} \\
& \sim\left(\sum_{i=1}^{n} a_{i}^{4}\right)^{1 / 2}=\left(\sum_{i=1}^{n} \frac{1}{i}\right)^{1 / 2} .
\end{aligned}
$$


We can now apply the same arguments as in the proof of Theorem 2.14 and obtain

$$
\left\|\sum b_{j} f^{\left(-3^{j}\right)}\right\|_{p} \sim\left\|\sum b_{j} f_{0}^{\left(-3^{j}\right)}\right\|_{p} \vee\left(\sum\left|b_{j}\right|^{p}\right)^{1 / p}
$$

From this expression it follows that $\left(f^{\left(-3^{j}\right)}\right)_{j=1}^{\infty}$ is basic.

Indeed

$$
\left\|\sum_{j=1}^{n} b_{j} f^{\left(-3^{j}\right)}\right\|_{p} \sim\left(\sum_{j=1}^{n}\left(b_{j} a_{j-1}-b_{j-1} a_{j}\right)^{2}+\left(b_{n} a_{n+1}\right)^{2}\right)^{1 / 2} \vee\left(\sum_{j=1}^{n}\left|b_{j}\right|^{p}\right)^{1 / p} .
$$

Let the right hand expression be equal to 1 with

$$
\sum_{j=1}^{n}\left(b_{j} a_{j-1}-b_{j-1} a_{j}\right)^{2}+\left(b_{n} a_{n+1}\right)^{2}=1 .
$$

Then if $\left(b_{n} a_{n+1}\right)^{2} \leq 1 / 2$, for any extension $\left(b_{i}\right)_{i=1}^{m}, m>n$, the right hand expression is at least $1 / \sqrt{2}$. If $\left(b_{n} a_{n+1}\right)^{2} \geq 1 / 2$, then $b_{n}>1 / 2^{1 / 4}$, and so $\left(\sum_{j=1}^{m}\left|b_{j}\right|^{p}\right)^{1 / p} \geq$ $2^{-1 / 4}$.

Finally $\left(f^{\left(-3^{j}\right)}\right)_{j=1}^{\infty}$ is not unconditional since

$$
\left\|\sum_{j=1}^{n} a_{j} f^{\left(-3^{j}\right)}\right\|_{p} \sim(\log n)^{1 / p}
$$

while

$$
\left\|\sum_{j=1}^{n}(-1)^{j+1} a_{j} f^{\left(-3^{j}\right)}\right\|_{p} \sim(\log n)^{1 / 2}
$$

The translation problem can, of course, be considered in other rearrangement invariant function spaces on $\mathbb{R}$. We end this section with a simple result in the space $L_{p}(\mathbb{R}) \cap L_{2}(\mathbb{R})$ for $2<p<\infty$. The norm is given by $\|f\|=\|f\|_{p} \vee\|f\|_{2}$ and the space is isomorphic to $L_{p}(\mathbb{R})$ (see, e.g., [JMST] for more on this space).

Proposition 2.18. Let $2<p<\infty$ and let $\left(f_{i}\right)_{i=1}^{\infty}$ be an unconditional basis for $X \subseteq L_{p}(\mathbb{R}) \cap L_{2}(\mathbb{R})$ consisting of translations of some fixed $f \in L_{p}(\mathbb{R}) \cap L_{2}(\mathbb{R})$. Then $\left(f_{i}\right)_{i=1}^{\infty}$ is equivalent to the unit vector basis of $\ell_{2}$.

Proof. As before, by first carefully approximating in both $\|\cdot\|_{p}$ and $\|\cdot\|_{2}$ each $f_{i}$ by a simple dyadic function $\tilde{f}_{i}$ and then choosing a block basis $\left(g_{i}\right)_{i=1}^{\infty}$ of $\left(h_{(i, n)}\right)$ with $\left|g_{i}\right|=\left|\tilde{f}_{i}\right|$ for all $i$, we obtain that $\left(g_{i}\right)_{i=1}^{\infty}$ is equivalent to $\left(f_{i}\right)_{i=1}^{\infty}$ in $L_{p}(\mathbb{R}) \cap L_{2}(\mathbb{R})$.

Now $\left(g_{i}\right)_{i=1}^{\infty}$ is unconditional and semi-normalized in $L_{p}(\mathbb{R}) \cap L_{2}(\mathbb{R})$ which is isomorphic to $L_{p}$. Hence by (1.2), $\left(g_{i}\right)$ admits an upper $\ell_{2}$-estimate. Furthermore $\left(g_{i}\right)_{i=1}^{\infty}$ is unconditional and semi-normalized in $L_{2}(\mathbb{R})$ and thus also admits a lower $\ell_{2}$-estimate in $\|\cdot\|_{2}$ and so in $L_{p}(\mathbb{R}) \cap L_{2}(\mathbb{R})$.

\section{Discrete Versions of the PRoblem}

It remains open if $L_{p}(\mathbb{R}), 1<p<\infty$, admits a basis of translations of some fixed $f \in L_{p}(\mathbb{R})$ (see section 4 for more open problems). The examples in section 3 were all integer translations and this leads to a natural

Question 3.1. Let $1<p<\infty$. Is there a set $\Lambda=\left\{\lambda_{n}: n \in \mathbb{N}\right\} \subseteq \mathbb{Z}$ and an $f \in L_{p}(\mathbb{R})$ so that that $\left(f_{\left(\lambda_{n}\right)}: n \in \mathbb{N}\right)$ is a basis for $L_{p}(\mathbb{R})$ ? 
The answer is no for $1<p \leq 2$ (and of course for $p=1$ by Theorem 1.7]) by Theorem 1.3. We also deduce this as a corollary to Proposition 3.7 below. The answer is also no for $\Lambda=\mathbb{Z}$ by $\mathrm{AO}$.

Proposition $3.2([\mathrm{AO}])$. Let $1<p<\infty$. There is no $\lambda>0$, and $f \in L_{p}(\mathbb{R})$ so that $\left\{f_{(\lambda n)}: n \in \mathbb{Z}\right\}$ can be ordered to be a basis for $L_{p}(\mathbb{R})$.

Proof. We will prove a more general result below (see Proposition 3.7 and Corollary 3.12 .

We can do a bit better in $L_{1}$ for certain spaces $X_{1}\left(f,(\lambda n)_{n \in \mathbb{Z}}\right)$. By Theorem 1.1. $X_{1}(f, \mathbb{R})=L_{1}(\mathbb{R})$ forces $\hat{f}(t) \neq 0$ for all $t$.

Lemma 3.3. Let $f \in L_{1}(\mathbb{R})$ with $\widehat{f}(t) \neq 0$ for all $t$, and let $\Lambda=\left\{\lambda_{n}: n \in \mathbb{N}\right\}$ be uniformly discrete. Then $\left\{f_{\left(\lambda_{n}\right)}\right\}_{n \in \mathbb{N}}$ is a non-fundamental minimal system in $L_{1}(\mathbb{R})$.

Proof. We use the fact that for a uniformly discrete $\Lambda$, there exists a $>0$ so that $\left(e^{i \lambda_{n} t}\right)_{n \in \mathbb{N}}$ is not complete in $C[-a, a]$. As pointed out to us by J. Bruna, this follows from the Paley-Wiener theory by constructing, from an entire function of finite exponential type, a Paley-Wiener function which vanishes on $\Lambda$. Alternately, this can also be quickly deduced from the Beurling-Malliavin radius of completeness formula (cf. [Ko, section IX D]) and the fact that the uniformly discrete sequences have finite Beurling-Malliavin density. For convenience of the reader, we present a proof. We recall the definition of Beurling-Malliavin density $D_{B M}$. For $\Lambda \subset(0, \infty)$ and $D>0$, a family of disjoint intervals $\left(a_{k}, b_{k}\right), 0<a_{1}<b_{1}<\cdots<a_{k}<b_{k}<$ $\ldots \nearrow \infty$ is called substantial for $D$ if

$$
\frac{n_{\Lambda}\left(a_{k}, b_{k}\right)}{b_{k}-a_{k}}>D, k=1,2, \ldots, \quad \sum_{k}\left(\frac{b_{k}-a_{k}}{b_{k}}\right)^{2}=\infty
$$

where $n_{\Lambda}\left(a_{k}, b_{k}\right)$ is the number points of $\Lambda$ in the interval $\left(a_{k}, b_{k}\right)$. Then the density is defined by

$$
D_{B M}(\Lambda)=\sup \{D>0 \text { : there exists a substantial family for } D\} .
$$

For a general $\Lambda$, put $D_{B M}(\Lambda)=\max \left\{D_{B M}\left(\Lambda^{+}\right), D_{B M}\left(\Lambda^{-}\right)\right\}$where $\Lambda^{+}=\Lambda \cap \mathbb{R}^{+}$, $\Lambda^{-}=(-\Lambda) \cap \mathbb{R}^{+}$. The Beurling-Malliavin radius of completeness theorem asserts that $\left\{e^{i \lambda_{n} t}: \lambda_{n} \in \Lambda\right\}$ is complete in $C[-a, a]$ if and only if $\pi D_{B M}(\Lambda) \geq a$.

Now suppose that $\Lambda$ is uniformly discrete, and let $\delta=\inf \left\{\left|\lambda-\lambda^{\prime}\right|: \lambda, \lambda^{\prime} \in \Lambda, \lambda \neq\right.$ $\left.\lambda^{\prime}\right\}>0$. Since $n_{\Lambda}\left(a_{k}, b_{k}\right) /\left(b_{k}-a_{k}\right)<2 / \delta$ for all $b_{k}>a_{k}>0$, no $D>2 / \delta$ can be substantial for $\Lambda$, and therefore $D_{B M}(\Lambda)<2 / \delta$. Thus, by the Beurling-Malliavin theorem, $\left(e^{i \lambda_{n} t}\right)_{n \in \mathbb{N}}$ is not complete in $C[-b, b]$ for $b>2 / \delta$.

To see the minimality of $\left\{f_{\left(\lambda_{n}\right)}\right\}$, suppose to the contrary that for some $n_{0}$, $f_{\lambda_{n_{0}}} \in\left[\left(f_{\lambda_{n}}\right)_{n \neq n_{0}}\right]$ in $L_{1}(\mathbb{R})$. Then $\hat{f}_{\lambda_{n_{0}}}(t)=\hat{f}(t) e^{-i \lambda_{n_{0}} t} \in\left[\left(\hat{f}(t) e^{-i \lambda_{n} t}\right)_{n \neq n_{0}}\right]$ in $C_{0}(\mathbb{R})$. Now $\hat{f}(t) \neq 0$ for all $t$, so $e^{-i \lambda_{n_{0}} t} \in\left[\left\{e^{-i \lambda_{n} t}: n \neq n_{0}\right\}\right] \subset C[-b, b]$ for all $b>0$. Thus $\left(e^{-i \lambda_{n} t}\right)_{n \neq n_{0}}$ is complete in $C[-b, b]$ (cf. [Yo, Theorem 8, p. 129]). This contradicts the fact when $b>a$. Similarly, observe that $\left\{f_{\left(\lambda_{n}\right)}\right\}$ cannot be fundamental in $L_{1}(\mathbb{R})$, indeed otherwise $\left(e^{-i \lambda_{n} t}\right)_{n \in \mathbb{N}}$ would be complete in $C[-b, b]$ for all $b>0$.

Note that the assumption $\hat{f}(t) \neq 0$ for all $t$ is not frivolous due to Remark $2.8 \mathrm{~b})$. 
Proposition 3.4. Let $f \in L_{1}(\mathbb{R})$ with $\hat{f}(t) \neq 0$ for all $t$, and let $\lambda>0$. Then $X_{1}\left(f,(\lambda n)_{n \in \mathbb{Z}}\right)$ embeds almost isometrically into $\ell_{1}$.

Proof. By Corollary 2.4 it suffices to show that $\left(f_{(\lambda n)}\right)_{n \in \mathbb{Z}}$ is a bounded minimal system. By Lemma 3.3 it is a minimal system. Let $g(f)=1, g\left(f_{(\lambda n)}\right)=0$ for $n \in \mathbb{Z} \backslash\{0\}, g \in L_{\infty}(\mathbb{R})$. Then $\left(f_{(\lambda n)}, g_{(\lambda n)}\right)_{n \in \mathbb{Z}}$ is a bounded minimal system.

Proposition 3.2 generalizes to $\ell_{p}$-sums of a separable infinite dimensional Banach space $X$. Define $\ell_{p}(X)=\ell_{p}(\mathbb{Z}, X)=\left(\bigoplus_{n \in \mathbb{Z}} X\right)_{\ell_{p}}$. For $F=\left(f_{n}: n \in \mathbb{Z}\right) \in \ell_{p}(X)$ and $k \in \mathbb{Z}$, let $F^{(k)}$ be $F$ shifted right $k$ times. Precisely, $F^{(k)}=\left(f_{n-k}\right)_{n \in \mathbb{Z}}$.

Proposition 3.5. Let $X$ be a separable infinite dimensional Banach space, $1 \leq$ $p<\infty$. There does not exist $F \in \ell_{p}(\mathbb{Z}, X)$ so that $\left\{F^{(k)}: k \in \mathbb{Z}\right\}$ is a basis for $\ell_{p}(\mathbb{Z}, X)$ in some order.

Proof. Let $1 / p+1 / q=1$, and assume for some $F$ that $\left(F^{\left(n_{i}\right)}\right)_{i=1}^{\infty}$ is a basis for $\ell_{p}(\mathbb{Z}, X)$ where $\left(n_{i}\right)_{i=1}^{\infty}$ is a reordering of $\mathbb{Z}$. Let $\left(G_{i}\right)_{i \in \mathbb{N}} \subseteq \ell_{q}\left(\mathbb{Z}, X^{*}\right)$ be the biorthogonal functionals to $\left(F^{\left(n_{i}\right)}\right)_{i=1}^{\infty}$. Choose $i_{0}$ with $n_{i_{0}}=0$, and set $G_{i_{0}}=G=\left(g_{n}\right)_{n \in \mathbb{Z}}$, with $g_{n} \in X^{*}$ for $n \in \mathbb{N}$.

For $n, m \in \mathbb{Z}$,

$$
\left\langle F^{(n)}, G^{(m)}\right\rangle=\sum_{k \in \mathbb{Z}}\left\langle f_{k-n}, g_{k-m}\right\rangle=\sum_{k \in \mathbb{Z}}\left\langle f_{k+m-n}, g_{k}\right\rangle=\left\langle F^{(n-m)}, G_{i_{0}}\right\rangle=\delta_{(m, n)} .
$$

Again, from the uniqueness of the biorthogonal functionals to a basis (for $\left.\ell_{p}(\mathbb{Z}, X)\right)$, we see that $G_{i}=G^{\left(n_{i}\right)}$ for all $i \in \mathbb{N}$.

Choose $j \in \mathbb{N}$ with

$$
\left(\sum_{i=j+1}^{\infty}\left\|f_{-n_{i}}\right\|^{p}\right)^{1 / p} \leq \frac{1}{2\|G\|} .
$$

Since $X$ is infinite dimensional, there exists $x \in S_{X}$ with $g_{-n_{i}}(x)=0$ for all $i \leq j$. Set $H=\left(\delta_{(0, n)} x: n \in \mathbb{Z}\right) \in \ell_{p}(\mathbb{Z}, X)$. Then

$$
H=\sum_{i=1}^{\infty}\left\langle H, G^{\left(n_{i}\right)}\right\rangle F^{\left(n_{i}\right)}=\sum_{i=j+1}^{\infty}\left\langle H, G^{\left(n_{i}\right)}\right\rangle F^{\left(n_{i}\right)} .
$$

Hence,

$$
\begin{gathered}
1=\|x\|=\|H\|=\left\|\sum_{i=j+1}\left\langle H, G^{\left(n_{i}\right)}\right\rangle F^{\left(n_{i}\right)}\right\|=\sum_{i=j+1}^{\infty}\left\langle g_{-n_{i}}, f_{-n_{i}}\right\rangle \\
\leq \sum_{i=j+1}^{\infty}\left\|g_{-n_{i}}\right\|\left\|f_{-n_{i}}\right\| \leq\|G\|\left(\sum_{i=j+1}^{\infty}\left\|f_{-n_{i}}\right\|^{p}\right)^{1 / p} \leq \frac{1}{2},
\end{gathered}
$$

a contradiction.

Problem 3.6. Let $2<p<\infty$, and let $X$ be a Banach space with $\operatorname{dim} X \geq 2$. Does there exist $F \in \ell_{p}(\mathbb{Z}, X)$ and $\left(\lambda_{i}: i \in \mathbb{N}\right) \subseteq \mathbb{Z}$ so that $\left(F^{\left(\lambda_{i}\right)}\right)_{i=1}^{\infty}$ is a basis for $\ell_{p}(\mathbb{Z}, X)$ ? What if $\operatorname{dim} X=2$ or if $X=\ell_{p}$ ?

We do not ask the question for $p \leq 2$ because of the following proposition which generalizes Proposition 3.5 in that case. 
Proposition 3.7. Let $1 \leq p \leq 2$, and let $X$ be a Banach space with $\operatorname{dim}(X) \geq 2$. Let $F=\left(f_{i}: i \in \mathbb{Z}\right) \in \ell_{p}(\mathbb{Z}, X)$. Then $\left[\left\{F^{(n)}: n \in \mathbb{Z}\right\}\right] \neq \ell_{p}(\mathbb{Z}, X)$.

Corollary $3.8([\mathrm{AO}])$. Let $1<p \leq 2, f \in L_{p}(\mathbb{R})$, and $\lambda>0$. Then $\left.\left[f_{(\lambda n)}: n \in \mathbb{Z}\right\}\right]$ is a proper subspace of $L_{p}(\mathbb{R})$. In particular, no subsequence of $\left\{f_{(\lambda n)}: n \in \mathbb{Z}\right\}$ can be ordered to form a basis for $L_{p}(\mathbb{R})$.

Proof. We let $F$ denote the Fourier transform on $L_{1}(\mathbb{R})+L_{2}(\mathbb{R})$ into the space of measurable functions on $\mathbb{R}$. $F$ is a bounded linear operator, restricted to $L_{1}(\mathbb{R})$ (into $C_{0}(\mathbb{R})$ ) and when restricted to $L_{2}(\mathbb{R})$ (into $L_{2}(\mathbb{R})$ ). By the Riesz-Thorin interpolation theorem, $F$ is also bounded as a linear operator from $L_{p}(\mathbb{R})$ into $L_{q}(\mathbb{R})(1 / p+1 / q=1)$. Now since $L_{1}(\mathbb{R}) \cap L_{2}(\mathbb{R}) \subseteq L_{p}(\mathbb{R}), F\left(L_{p}(\mathbb{R})\right)$ is dense in $L_{q}(\mathbb{R})$. For $f \in L_{p}(\mathbb{R})$ and $s \in \mathbb{R}$, we have $F\left(f_{s}\right)=e^{-i s(\cdot)} F(f)$. Indeed for $f \in L_{1}(\mathbb{R})$ and $t \in \mathbb{R}$,

$$
\begin{aligned}
F\left(f_{s}\right)(t) & =\frac{1}{\sqrt{2 \pi}} \int_{-\infty}^{\infty} e^{i x t} f(x-s) d x \\
& =\frac{1}{\sqrt{2 \pi}} \int_{-\infty}^{\infty} e^{-i(u+s) t} f(u) d x=e^{-i s} F(f)(t) .
\end{aligned}
$$

For a general $f \in L_{p}(\mathbb{R})$ the result follows by the standard density argument.

Let $f \in L_{p}(\mathbb{R})$ and $\lambda \in \mathbb{R}$. If $\left[\left\{f_{(\lambda n)}: n \in \mathbb{Z}\right\}\right]=L_{p}(\mathbb{R})$, then $\left[\left\{e^{i n \lambda(\cdot)} F(f)\right.\right.$ : $n \in \mathbb{Z}\}]=L_{q}(\mathbb{R})$. This implies that $F(f) \neq 0$ a.e., and that $\left[\left\{e^{i n \lambda(\cdot)}: n \in \mathbb{Z}\right\}\right]=$ $L_{q}\left(|F(f)|^{q} d x\right)$. This in turn implies that all elements $g$ of $L_{p}\left(|F(f)|^{q} d x\right)$ are $\lambda$ periodic $(g(x)-g(x+\lambda)=0$ a.e. $)$, a contradiction.

Remark 3.9. For $2<p<\infty$, it is shown in $\mathrm{AO}$ (Theorem 1.2 above) that there exists $f \in L_{p}(\mathbb{R})$ so that $\left[\left(f^{(n)}\right)_{n \in \mathbb{Z}}\right]=L_{p}(\mathbb{R})$.

We will use the Fourier transform on the abelian group $\mathbb{Z}$ (see $[\mathrm{Ru}$ ) and also assume our spaces to be defined over the complex field. For $x=\left(\xi_{j}\right) \in \ell_{1}(\mathbb{Z})$, we let $\widehat{x}$ be the function

$$
\widehat{x}:[-\pi, \pi] \rightarrow \mathbb{R}, \quad \widehat{x}(t)=\sum_{n \in \mathbb{Z}} \xi_{n} e^{i n t} .
$$

It is easy to see that $\widehat{x} \in C(T)$ when $x \in \ell_{1}(\mathbb{Z})$ (identifying, as usual, the torus $T$ with $[-\pi, \pi]$ by identifying $\pi$ and $-\pi)$. Also the map

$$
\widehat{(\cdot)}: \ell_{1}(\mathbb{Z}) \rightarrow C(T), \quad x \rightarrow \hat{x},
$$

is a bounded linear operator of norm 1 . For any $x=\left(\xi_{n}: n \in \mathbb{Z}\right)$,

$$
\|\hat{x}\|_{2}^{2}=\int_{-\pi}^{\pi}\left|\sum_{n \in \mathbb{Z}} \xi_{n} e^{i n t}\right|^{2} d t=\int_{-\pi}^{\pi} \sum_{m, n \in \mathbb{Z}} \bar{\xi}_{m} \xi_{n} e^{i(n-m) t} d t=2 \pi \sum_{n \in \mathbb{Z}}\left|\xi_{n}\right|^{2} .
$$

Thus $\widehat{(\cdot)}$ extends to an isometry from $\ell_{2}(\mathbb{Z})$ to $L_{2}\left(T, \frac{1}{2 \pi} d x\right)$.

Again, by the Riesz-Thorin interpolation theorem, the Fourier transform is a bounded linear operator from $\ell_{p}(\mathbb{Z})$ into $L_{q}(T)$ for $1 \leq p \leq 2,1 / p+1 / q=1$.

Since $\left\{\widehat{x}: x \in \ell_{1}(\mathbb{Z})\right\}$ is dense in $L_{2}(T)$, it follows that the image under the Fourier transform of $\ell_{p}(\mathbb{Z})$ is dense in $L_{q}(T)$. 
We also need two lemmas before proving Proposition 3.7 The first is an easy exercise in real analysis.

Lemma 3.10. Let $\nu \ll \mu$ be two $\sigma$-finite measures on the measure space $(\Omega, \Sigma)$. Then for $1 \leq p<\infty$, if $D \subseteq L_{p}(\nu) \cap L_{p}(\mu)$ is dense in $L_{p}(\mu)$, it is also dense in $L_{p}(\nu)$.

Proof. Let $\rho$ be the Radon-Nikodym density of $\nu$ with respect to $\mu$. For $n \in \mathbb{N}$ set

$$
A_{n}=\left\{\omega \in \Omega: \frac{1}{n} \leq \rho(\omega) \leq n\right\} .
$$

For $n \in \mathbb{N}$, it follows that $L_{p}\left(\left.\mu\right|_{A_{n}}\right)=L_{p}\left(\left.\nu\right|_{A_{n}}\right)$. Also by canonically identifying $L_{p}\left(\left.\nu\right|_{A_{n}}\right)$ with a subspace of $L_{p}(\nu), \bigcup_{n \in \mathbb{N}} L_{p}\left(\left.\nu\right|_{A_{n}}\right)$ is dense in $L_{p}(\nu)$, and this yields the results.

Lemma 3.11. Let $1 \leq p \leq 2$, and let $x=\left(\xi_{n}: n \in \mathbb{Z}\right) \in \ell_{p}(\mathbb{Z})$. Then $\left[\left(x^{(2 n)}\right)_{n \in \mathbb{Z}}\right] \neq \ell_{p}(\mathbb{Z})$.

Proof. Recall $x^{(n)}=\left(\xi_{j-n}: j \in \mathbb{Z}\right)$, for $n \in \mathbb{Z}$. For $n \in \mathbb{N}, t \in T$ and $z=\left(\zeta_{j}: j \in\right.$ $\mathbb{Z}) \in \ell_{1}(\mathbb{Z})$ we have

$$
\widehat{z^{(n)}}(t)=\sum_{j \in \mathbb{Z}} \zeta_{j-n} e^{i j t}=\sum_{\ell \in \mathbb{Z}} \zeta_{\ell} e^{i(\ell+n) t}=e^{i n t} \widehat{z} .
$$

By a density argument, we see that for any $x \in \ell_{p}(\mathbb{Z})$ and $n \in \mathbb{Z}, \widehat{x^{(n)}}=e^{i n(\cdot)} \widehat{x}$.

Assume, to the contrary, that $\left[\left(x^{(2 n)}\right)_{n \in \mathbb{Z}}\right]=\ell_{p}(\mathbb{Z})$. It then follows that $\left[\left\{e^{i 2 n(\cdot)} \widehat{x}: n \in \mathbb{Z}\right\}\right]=L_{q}(T)$ and thus $\widehat{x} \neq 0$ a.e. Also that

$$
\left[\left\{e^{i 2 n(\cdot)}: n \in \mathbb{Z}\right\}\right]=L_{q}\left(T,|\widehat{x}|^{q} d t\right) .
$$

By Lemma 3.10, this implies that

$$
\left[\left\{e^{i 2 n(\cdot)}: n \in \mathbb{Z}\right\}\right]=L_{q}(T) .
$$

Since for any $n \in \mathbb{Z}$,

$$
\begin{aligned}
& 2 \pi\left\langle e^{i 2 n(\cdot)}, \chi_{[-\pi, 0]}-\chi_{[0, \pi]}\right\rangle=\int_{-\pi}^{0} e^{i 2 n t} d t-\int_{0}^{\pi} e^{i 2 n t} d t \text { if } n=0, \\
&=\int_{0}^{\pi}\left(e^{-i 2 n t}-e^{i 2 n t}\right) d t= \begin{cases}0, & \text { if } n \neq 0, \\
-2 \int_{0}^{\pi} \sin (2 n t) d t=0,\end{cases}
\end{aligned}
$$

this cannot be true.

Proof of Proposition 3.7. After projecting $X$ onto $\ell_{p}^{2}$, we see that we may assume $X=\ell_{p}^{2}$. Let $I$ be the obvious isometry between $\ell_{p}(\mathbb{Z}, X)$, and let $\ell_{p}(\mathbb{Z})$ be denoted

$$
\left(x_{j}\right)_{j \in \mathbb{Z}} \longmapsto\left(y_{j}\right)_{j \in \mathbb{Z}},
$$

where if $x_{j}=\left(x_{(j, 1)}, x_{(j, 2)}\right) \in \ell_{p}^{2}$, then

$$
y_{2 j}=x_{(j, 1)}, \quad y_{2 j+1}=x_{(j, 2)} .
$$

Then for $\left(x_{j}\right)_{j \in \mathbb{Z}} \in \ell_{p}\left(\ell_{p}^{2}\right),\left(x^{(n)}\right)_{n \in \mathbb{Z}}=\left(I(x)^{2 n}\right)_{n \in \mathbb{Z}}$ and the result follows from Lemma 3.11 
Remark. As noted above by the results of $[\mathrm{AO}$ in section 4 we cannot hope to prove that given $f \in L_{p}(\mathbb{R}), 2<p<\infty,\left[\left(f^{(n)}\right)_{n \in \mathbb{Z}}\right] \neq L_{p}(\mathbb{R})$. Nevertheless, by dualizing Proposition 3.7, we have the following

Corollary 3.12. Let $X$ be a Banach space with $\operatorname{dim}(X) \geq 2$, and let $2 \leq p<\infty$. Let $F=\left(f_{i}\right)_{i \in \mathbb{Z}} \in \ell_{p}(\mathbb{Z}, X)$. Then $\left\{F^{(n)}: n \in \mathbb{Z}\right\}$ is not a basis for $\ell_{p}(\mathbb{Z}, X)$ under any ordering.

Proof. Assume that $F \in\left(f_{i}\right)_{i \in \mathbb{Z}} \in \ell_{p}(\mathbb{Z}, X)$ and that $\left(n_{s}\right)_{s \in \mathbb{N}}$ is an ordering of $\mathbb{Z}$ so that $\left(F^{\left(n_{s}\right)}\right)_{s=1}^{\infty}$ is a basis for $\ell_{p}(\mathbb{Z}, X)$. Let $\left(G_{s}\right)_{s=1}^{\infty} \subseteq \ell_{q}\left(\mathbb{Z}, X^{*}\right)$ be the biorthogonal functionals of $\left(F^{\left(n_{s}\right)}\right)_{s=1}^{\infty}$. Set $G=\left(g_{j}\right)_{j \in \mathbb{Z}}=G_{1}$. We let $G^{(m)}=\left(g_{j-m}\right)_{j \in \mathbb{Z}}$, as usual. For $s, t \in \mathbb{N}$ and $m \in \mathbb{Z}$, we have

$$
\begin{aligned}
\left\langle F^{\left(n_{s}\right)}, G^{\left(n_{t}\right)}\right\rangle & =\sum_{j \in \mathbb{Z}}\left\langle f_{j-n_{s}}, g_{j-n_{t}}\right\rangle=\sum_{k \in \mathbb{Z}}\left\langle f_{k+n_{t}-n_{s}}, g_{k}\right\rangle \\
& =\left\langle F^{\left(n_{s}-n_{t}\right)}, G_{1}\right\rangle= \begin{cases}1, & \text { if } n_{s}-n_{t}=n_{1}, \\
0, & \text { if } n_{s}-n_{t} \neq n_{1} .\end{cases}
\end{aligned}
$$

As before, we see that $G_{s}=G^{\left(n_{s}-n_{1}\right)}$. In particular, $\operatorname{span}\left\{G^{(n)}: n \in \mathbb{Z}\right\}$ is $w^{*}$-dense in $\ell_{q}\left(X^{*}\right)$. Let $E$ be a two-dimensional subspace of $X$, and let $P$ be a projection of $X$ onto $E$. Let $Q: \ell_{p}(\mathbb{Z}, X) \rightarrow \ell_{p}(\mathbb{Z}, E)$ be the projection given by $Q(H)=\left(P\left(h_{i}\right)\right)_{i \in \mathbb{Z}}$. It follows that $\operatorname{span}\left(\left.G^{(n)}\right|_{\ell_{p}(\mathbb{Z}, E)}\right)_{n \in \mathbb{Z}}$ is $w^{*}$-dense in $\ell_{q}\left(\mathbb{Z}, E^{*}\right)$ and hence norm dense (the latter is reflexive). This contradicts Proposition 3.7 .

\section{Results from the Literature AND open Problems}

We first cite some more known results from the literature.

Theorem 4.1 ([DH, Theorem 5.1(b)]). Let $g^{(1)}, g^{(2)}, \ldots, g^{(m)} \in L_{2}\left(\mathbb{R}^{d}\right) \cap L_{1}\left(\mathbb{R}^{d}\right)$, and let $\Gamma_{1}, \Gamma_{2}, \ldots, \Gamma_{m} \subset \mathbb{R}^{d}$ be countable. Then $\left\{g_{(\lambda)}^{(i)}: i=1,2, \ldots, m, \lambda \in \Gamma_{i}\right\}$ cannot be ordered to be a Schauder basis of $L_{2}\left(\mathbb{R}^{d}\right)$.

Theorem 4.2 ([ER] and [Ro, cf. [H, Theorem 9.18]). If $g \in L_{p}\left(\mathbb{R}^{d}\right), g \neq 0$, and $1 \leq p \leq \frac{2 d}{d-1}$, then the functions $\left\{g\left((\cdot)-\alpha_{k}\right): k=1,2, \ldots, N\right\}$ are linearly independent for any $N \in \mathbb{N}$ and any collection $\left(\alpha_{k}\right)_{k=1}^{N} \subseteq \mathbb{R}^{d}$ of distinct points.

If $\frac{2 d}{d-1}<p \leq \infty$, then for $N \in \mathbb{N}$ there exists $0 \neq g \in L_{p}\left(\mathbb{R}^{d}\right)$ and distinct points $\left(\alpha_{k}\right)_{k=1}^{N} \subseteq \mathbb{R}^{d}$ so that $\left\{g\left((\cdot)-\alpha_{k}\right): k=1,2, \ldots, N\right\}$ is linearly dependent.

Our last cited result requires some notation. For $\Lambda \subseteq \mathbb{R}$, let $\mathcal{E}(\Lambda)=\operatorname{span}\left\{e^{i \lambda(\cdot)}\right.$ : $\lambda \in \Lambda\}$. Let $R(\Lambda)=\sup \{\rho>0: \mathcal{E}(\Lambda)$ is dense in $C[-\rho, \rho]\}$. Recall, $\Lambda \subseteq \mathbb{R}$ is discrete if it has no accumulation points.

Theorem 4.3 ([BOU, Theorem 1$])$. Let $\Lambda \subseteq \mathbb{R}$ be discrete. There exists $f \in L_{1}(\mathbb{R})$ so that $\left[\left\{f_{(\lambda)}: \lambda \in \Lambda\right\}\right]=L_{1}(\mathbb{R})$ if and only if $R(\Lambda)=\infty$.

Finally we list some problems that remain open. The main one is

Problem 4.4. Let $1<p<\infty$. Does there exist $f \in L_{p}(\mathbb{R})$ and $\Lambda \subseteq \mathbb{R}$ so that $\left\{f_{(\lambda)}: \lambda \in \Lambda\right\}$ can be ordered to be a basis for $L_{p}(\mathbb{R})$ ? Can we find $f$ and a uniformly discrete set $\Lambda$ so that $\left\{f_{(\lambda)}: \lambda \in \Lambda\right\}$ can be ordered to be a frame for $L_{p}(\mathbb{R})$ ? 
By identifying $L_{p}(\mathbb{R})$ with $L_{p}[0,1]$ we have a more general version of the basis problem in 4.4 .

Problem 4.5. Does there exist a normalized basis $\left(f_{n}\right)_{n=1}^{\infty}$ for $L_{p}[0,1], 1<p<\infty$, so that for all $0<b<1$,

$$
\sum_{n=1}^{\infty}\left\|\left.f_{n}\right|_{[0, b]}\right\|^{p}<\infty ?
$$

If $4<p<\infty$, can we find such $f_{n}$ 's which form an unconditional basis for $L_{p}[0,1]$ ?

Problem 4.6. Let $4<p<\infty$. Does there exist $f \in L_{p}(\mathbb{R})$ and $\Lambda \subseteq \mathbb{R}$ so that $\left(f_{(\lambda)}\right)_{\lambda \in \Lambda}$ is an unconditional basis for $L_{p}(\mathbb{R})$ ?

We can also raise questions asking for less, and here is one such question.

Problem 4.7. Let $1<p<4$. Does there exist $f \in L_{p}(\mathbb{R})$ and a uniformly discrete set $\Lambda \subseteq \mathbb{R}$ so that $\left[\left\{f_{(\lambda)}: \lambda \in \Lambda\right\}\right] \subseteq L_{p}(\mathbb{R})$ contains an isomorph of $\ell_{2}$ and $\left(f_{(\lambda)}\right)_{\lambda \in \Lambda}$ can be ordered to be a basic sequence (or a frame)?

Problem 4.8. Let $\Lambda \subseteq \mathbb{R}$ be uniformly discrete and $f \in L_{1}(\mathbb{R})$. Does $X_{1}(f, \Lambda)$ embed into $\ell_{1}$ ?

\section{REFERENCES}

[AOd] D. Alspach and E. Odell, " $L_{p}$ spaces", in Handbook of Geometry of Banach Spaces, Vol. 1, W.B. Johnson and J. Lindenstrauss, eds., Elsevier, Amsterdam (2001), 123-159. MR.1863691 (2003b:46013)

[AO] A. Atzmon and A. Olevskii, Completeness of integer translates in function spaces on $\mathbb{R}$, J. of Approx. Theory 87 (1996), 291-327. MR1420335 (98a:46035)

[BOU] J. Bruna, A. Olevskii and A. Ulanovskii, Completeness in $L_{1}(\mathbb{R})$ of discrete translates, Rev. Mat. Iberoamericana 22 no. 1, (2006), 1-16. MR2267311 (2007k:42015)

[CDOSZ] P. G. Casazza, S. J. Dilworth, E. Odell, Th. Schlumprecht and A. Zsák, Coefficient quantization for frames in Banach spaces, J. Math. Anal. Appl. 348 (2008), 66-86. MR2449328 (2009g:46018)

[CHL] P. G. Casazza, D. Han and D. R. Larson, Frames for Banach spaces, the functional and harmonic analysis of wavelets and frames, (San Antonio, TX, 1999), Contemp. Math. 247 (1999), 149-182. MR1738089 (2000m:46015)

[DH] B. Deng and C. Heil, "Density of Gabor Schauder bases", in Wavelet Applications in Signal and Image Processing VIII (San Diego, CA, 2000), A. Aldroubi, A. Lane, and M. Unser, eds., Proc. SPIE 4119, SPIE, Bellingham, WA, (2000), 153-164.

[ER] G. Edgar and J. Rosenblatt, Difference equations over locally compact abelian groups, Trans. Amer. Math. Soc. 253 (1979), 273-289. MR536947 (80i:39001)

$[\mathrm{H}] \quad$ C. Heil, "Linear independence of finite Gabor systems" in Harmonic Analysis and Applications, A volume in honor of John J. Benedetto, Birkhauser, Boston, (2006), 171-206. MR 2249310 (2007d:42057)

$[\mathrm{J}] \quad$ W. B. Johnson, On quotients of $L_{p}$ which are quotients of $\ell_{p}$, Compositio Math. 34 (1) (1977), 69-89. MR0454595 (56:12844)

[JL] W. B. Johnson and J. Lindenstrauss, "Basic concepts in the geometry of Banach spaces", in Handbook of Geometry of Banach Spaces, Vol. 1, W. B. Johnson and J. Lindenstrauss, eds., Elsevier, Amsterdam (2001), 1-84. MR.1863689 (2003f:46013)

[JO] W. B. Johnson and E. Odell, Subspaces of $L_{p}$ which embed into $\ell_{p}$, Compos. Math. 28 (1974), 37-49. MR0352938 (50:5424)

[JMST] W. B. Johnson, B. Maurey, G. Schechtman and L. Tzafriri, Symmetric structures in Banach spaces, Mem. Amer. Math. Soc. 19 (217) (1979) v+298. MR.527010 (82j:46025)

[KP] M. I. Kadets and A. Pełczyński, Bases, lacunary sequences and complemented subspaces in the spaces $L_{p}$, Studia Math. 21 (1961/62), 161-176. MR0152879(27:2851)

$[\mathrm{KW}] \quad$ N. J. Kalton and D. Werner, Property $(M), M$-ideals, and almost isometric structure of Banach spaces, J. Reine Angew. Math. 461 (1995), 137-178. MR.1324212 (96m:46022) 
[Ko] P. Koosis, The logarithmic integral II, Cambridge Studies in Advanced Mathematics, 21. Cambridge University Press, Cambridge, 1992. MR.1195788 (94i:30027)

[LT] J. Lindenstrauss and L. Tzafriri, Classical Banach Spaces I: Sequence Spaces, Ergebnisse der Mathematik und ihrer Grenzgebiete 92, Springer-Verlag, Berlin (1977). MR0500056 (58:17766)

[Ol] A. Olevskii, Completeness in $L_{2}(\mathbb{R})$ of almost integer translates, C. R. Acad. Sci. Paris 324 (1997), 987-991. MR1451238 (98a:42002)

[OZ] T. E. Olson and R. A. Zalik, "Nonexistence of a Riesz basis of translates", Approximation Theory, Lecture Notes in Pure and Applied Math., 138, Dekker, New York (1992) 401-408. MR 1174120

[R] J. Rosenblatt, private communication.

[Ro] J. Rosenblatt, Linear independence of translations, J. Austral. Math. Soc. (Series A) 59 (1995), 131-133. MR1336456 (96f:42009)

[Ru] W. Rudin, Fourier Analysis on Groups, Interscience Publisher, John Wiley \& Sons (1967). MR0152834 (27:2808)

[S] G. Schechtman, A remark on unconditional basic sequences in $L_{p}(1<p<\infty)$, Israel J. Math. 19 (1974), 220-224. MR0511797 (58:23512)

[Wi] N. Wiener, The Fourier integral and certain of its applications, Cambridge University Press, Cambridge, 1933 reprint: Dover, New York, 1958. MR0100201 (20:6634)

[Yo] R. M. Young, An introduction to nonharmonic Fourier series, Pure and Applied Mathematics, 93. Academic Press, Inc., New York-London, 1980. MR591684 (81m:42027)

Department of Mathematics, The University of Texas at Austin, 1 University Station C1200, Austin, Texas 78712-0257

E-mail address: odell@math.utexas.edu

Department of Mathematics, University of North Texas, Denton, Texas 76203-5017

E-mail address: bunyamin@unt.edu

Department of Mathematics, Texas A\&M University, College Station, Texas 778433368

E-mail address: schlump@math.tamu.edu

Department of Mathematics, The University of Texas at Austin, 1 University Station C1200, Austin, Texas 78712-0257

E-mail address: btzheng@math.utexas.edu

Current address: Department of Mathematical Sciences, The University of Memphis, Memphis, Tennessee 38152-3240

E-mail address: bzheng@memphis.edu 